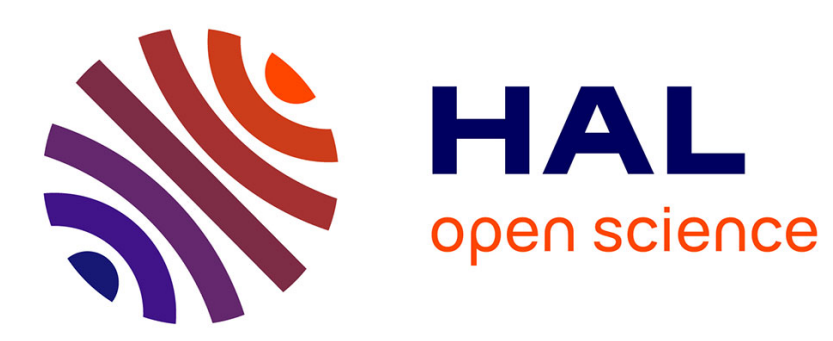

\title{
Reconstruction of the shape and surface impedance from acoustic scattering data for arbitrary cylinder
}

Jijun Liu, Gen Nakamura, Mourad Sini

\section{To cite this version:}

Jijun Liu, Gen Nakamura, Mourad Sini. Reconstruction of the shape and surface impedance from acoustic scattering data for arbitrary cylinder. 2007. hal-00136016

\author{
HAL Id: hal-00136016 \\ https://hal.science/hal-00136016 \\ Preprint submitted on 12 Mar 2007
}

HAL is a multi-disciplinary open access archive for the deposit and dissemination of scientific research documents, whether they are published or not. The documents may come from teaching and research institutions in France or abroad, or from public or private research centers.
L'archive ouverte pluridisciplinaire HAL, est destinée au dépôt et à la diffusion de documents scientifiques de niveau recherche, publiés ou non, émanant des établissements d'enseignement et de recherche français ou étrangers, des laboratoires publics ou privés. 


\title{
RECONSTRUCTION OF THE SHAPE AND SURFACE IMPEDANCE FROM ACOUSTIC SCATTERING DATA FOR ARBITRARY CYLINDER
}

\author{
J.J. LIU*, G. NAKAMURA ${ }^{\dagger}$, AND M. SINI $\ddagger$
}

\begin{abstract}
The inverse scattering for an obstacle $D \subset R^{2}$ with mixed boundary condition can be considered as a prototype for radar detection of complex obstacles with coated and non-coated parts of the boundary. We construct some indicator functions for this inverse problem using the far-field pattern directly, without the necessity to transform the far-field to the near field. Based on the careful singularity analysis, these indicator functions enable us to reconstruct the shape of the obstacle and distinguish the coated from the non-coated part of the boundary. Moreover, an explicit representation formula for the surface impedance in the coated part of the boundary is also given. Our reconstruction scheme reveals that the coated part of the obstacle is less visible than the non-coated one, which corresponds to the physical fact that the coated boundary absorbs some part of the scattered wave. Numerics are presented for the reconstruction formulas, which show that both the boundary shape and the surface impedance in the coated part of boundary can be reconstructed accurately. The theoretical reconstruction techniques proposed in this work can be applied in the practical 3-dimensional electromagnetic inverse scattering problems with hopeful numerical performances, which are of great importance in the design of non-detectable obstacles.
\end{abstract}

Key words. Inverse scattering, far-field, impedance boundary, singularity analysis, numerics.

AMS subject classifications. 35P25, 35R30, 78A45.

1. Introduction and examples. The inverse scattering problems aim to identify some properties of an obstacle such as the boundary shape and type from the information contained in the scattered wave for given incident waves. The optimization techniques are well-known for reconstructing the obstacle, up to some accuracy, by minimizing the objective functional for unknown obstacle from given inversion input data by iterations procedures. However, it seems that a good initial guess is needed.

In the recent years, some new inversion methods for the reconstruction of obstacle boundary have been proposed. The general and common idea of these methods is the construction of some indicator functions from given inversion input data, which depend on some detecting point (a parameter) varying inside or outside the obstacle. When this point approaches the obstacle, these indicator functions blowup. The linear sampling method [7], the factorization method [16] and the singular sources method [21] construct the indicator functions from the far-field pattern directly, while the probe method $[13,14]$ constructs the indicator in terms of the near field. The near field can be obtained from the far field by some regularization procedures [21]. However, we can also state the natural version of the probe method directly from the far field data, without reducing the far field to the near field, see [11]. For a review of these methods, the readers are referred to [22, 23] and for some relations between them to $[11,20]$.

If the scattering is caused by multiple obstacles with different types of boundary or with mixed boundary condition, one should identify both the boundary shape,

\footnotetext{
*Department of Mathematics, Southeast University, Nanjing, 210096, P.R. China (jjliu@seu.edu.cn).

†Department of Mathematics, Hokkaido University, Sapporo, 060-0810, Japan (gnaka@math.sci.hokudai.ac.jp).

$\ddagger$ Department of Mathematics, Yonsei University, Seoul, 120-749, Korea. New address: Ricam, Altenbergerstrasse 69, Linz, A-4040, Austria (mourad.sini@oeaw.ac.at).
} 
boundary type and the surface impedance. These kinds of problems come from some industry designs such as radar detection by electromagnetic wave scattering, see [10]. The obstacle is illuminated by an electromagnetic wave coming from an antenna. The wave is scattered by the obstacle and received by an antenna located in a different place. One of the objectives is to design the shape of the obstacle such that the reflected wave can be avoided or minimized in some directions. One possible way for this goal is to introduce a coating on the surface of the obstacle or on some of its parts. This is motivated by the fact that reflections are minimized by applying such a surface coating. The surface coating is modelled by introducing an impedance boundary condition on a part or on the whole surface of the scatterer, which gives a relation between the electric and the magnetic field through a coefficient called surface impedance.

Due to this practical importance, the reconstruction of boundary impedance has been addressed by many authors. In [1], the authors construct the inhomogeneous boundary impedance for a cylinder obstacle with known shape using only one incident wave, assuming that the surface impedance is distributed along the whole boundary of the obstacle. In this case, the scattering of electromagnetic waves can be described by the 2-dimensional Helmholtz equation. We also refer to [17], where an optimization method is applied. After reducing the far field to the near field, a moment method is suggested in [6] to reconstruct the surface impedance approximately in the case of completely coated obstacle, and the identification of different types of multiple obstacles is given in [5] in the case where on each obstacle we have one type of boundary condition.

The problem whether a part of the surface of the obstacle is coated or not is of relevant importance. Answering to this question and reconstructing the surface impedance, in case of coating, from far field measurements is our main object. In this work, we restrict ourself to the acoustic wave scattering governed by 2-dimensional Helmholtz equation, noticing that the 3 -dimensional electromagnetic wave scattering in the cylinder case can be modelled by the 2-dimensional Helmholtz equation [8]. These issues have been firstly considered in [2, 3], by the linear sampling method, where the authors simultaneously reconstruct the obstacle and compute the $L^{\infty}$-norm of the surface impedance. This can be used to answer the question of existence or absence of coating and give the value of the surface impedance in case it is known to be constant.

Motivated by these last works, our aim is to give another way to consider these issues and give further information on the obstacle. We proceed by constructing some indicator functions giving a direct link between the far-field pattern and the physical parameters of the obstacle. More precisely, we establish pointwise formulas which enable us to detect the boundary of the scatterer and distinguish and recognize the coated and the non-coated parts of the obstacle surface. In addition, on the coated part of the obstacle, the indicator functions give explicitly the pointwise values of this surface impedance as a functional of the far fields. These types of results have been initiated in [19], where the theoretical justification of these formulas in 3 -dimensional acoustic scattering is given. Since we need more singularity analysis in the 2-dimensional case than in 3-dimensional case, which is due to the use of a more singular point source, we give the theoretical justification of the steps where it is necessary and refer to [19] for the rest of proof. We would like to emphasize that we are reconstructing the obstacle, localizing the eventual coated part and reconstruct the surface impedance in on step, i.e. simultaneously, compare with $[[1],[2],[3],[5]$, 
[6], [17]]. Also, since the analysis is done pointwise, then we can also consider the multiple obstacles and give similar results.

The validity of the theoretical reconstruction formula presented in this paper is also checked by numerical tests with satisfactory performances. We would like to mention the following observations from the numerics. The coated part of an obstacle with larger impedance is less visible than the other part in terms of the value of the indicator. This explains the practical motivation for introducing the coating, i.e, to avoid or perturb the detection of an obstacle by applying the absorbing boundary layer. On the other hand, for non-convex obstacles with mixed boundary conditions, the inversion formulas proposed in this paper also generate a satisfactory reconstruction by combining different blowing-up criterion together. These reconstruction performances are supported by our numerical implementations given in the last section of this paper.

The rest of the paper is organized as follows. In section 1 , we state the problem mathematically. In section 2 , we present the results which we prove in section 3 . The section 4 is devoted to the numerical tests.

2. Statement of the problem. Let $D$ be a bounded domain of $R^{2}$ such that $R^{2} \backslash \bar{D}$ is connected. We assume that its boundary $\partial D$ is of class $C^{2}$ and has the following form

$$
\partial D=\overline{\partial D_{I}} \cup \overline{\partial D_{D}}, \quad \partial D_{I} \cap \partial D_{D}=\emptyset,
$$

where $\partial D_{D}$ and $\partial D_{I}$ are open surfaces in $\partial D$.

The propagation of time-harmonic acoustic fields in homogeneous cylinder media can be modeled by the Helmholtz equation

$$
\Delta u+\kappa^{2} u=0 \quad \text { in } \quad R^{2} \backslash \bar{D},
$$

where $\kappa>0$ is the wave number. At the part $\partial D_{I}$ of the obstacle boundary, we assume the total field $u$ to satisfy the impedance boundary condition while on the part $\partial D_{D}$ to satisfy the Dirichlet boundary condition. That is,

$$
\frac{\partial u}{\partial \nu}+i \kappa \sigma u=0 \text { on } \partial D_{I}
$$

with some impedance function $\sigma$ and

$$
u=0 \text { on } \partial D_{D},
$$

where $\nu$ is the outward unit normal of $\partial D$. We assume that $\sigma$ is a real valued Holder continuous function of order $\beta \in(0,1]$ and has a uniform lower bound $\sigma_{-}>0$ on $\partial D_{I}$. The part $\partial D_{I}$ is referred to by the coated part of $\partial D$ and $\partial D_{D}$ is the non-coated part.

For a given incident plane wave $u^{i}(x, d)=e^{i \kappa d \cdot x}$ with incident direction $d \in S^{1}$, where $S^{1}$ is a unit circle in $R^{2}$, we look for a solution $u:=u^{i}+u^{s}$ of (2.1), (2.2) and (2.3), where the scattered field $u^{s}$ satisfies the Sommerfeld radiation condition

$$
\lim _{r \rightarrow \infty} \sqrt{r}\left(\frac{\partial u^{s}}{\partial r}-i \kappa u^{s}\right)=0
$$

with $r=|x|$ and the limit is uniform for all directions $\hat{x} \in S^{1}$.

The mixed problem $(2.1)-(2.4)$ is well posed. More generally, for $f \in H^{\frac{1}{2}}\left(\partial D_{D}\right)$ and $h \in H^{-\frac{1}{2}}\left(\partial D_{I}\right)$, there exists a unique solution of the mixed problem 


$$
\left\{\begin{array}{l}
\left(\Delta+\kappa^{2}\right) u=0, \quad \text { in } R^{2} \backslash \bar{D} \\
u=f \text { on } \partial D_{D}, \\
\frac{\partial u}{\partial \nu}+i \kappa \sigma u=h, \quad \text { on } \partial D_{I}, \\
\lim _{r \rightarrow \infty} \sqrt{r}\left(\frac{\partial u}{\partial r}-i \kappa u\right)=0,
\end{array}\right.
$$

and the solution satisfies

$$
\|u\|_{H^{1}\left(\Omega_{R} \cap\left(R^{2} \backslash \bar{D}\right)\right)} \leq C_{R}\left(\|f\|_{H^{1 / 2}\left(\partial D_{D}\right)}+\|h\|_{H^{-\frac{1}{2}}\left(\partial D_{I}\right)}\right)
$$

where $\Omega_{R}$ is a disk of radius $R$ and $C_{R}$ is positive constant depending on $R$, see [4] for more details.

It is well known, see [8], that the scattered wave has the asymptotic behavior:

$$
u^{s}(x, d)=\frac{e^{i \kappa r}}{\sqrt{r}} u^{\infty}(\hat{x}, d)+O\left(r^{-3 / 2}\right), \quad r:=|x| \rightarrow \infty,
$$

where the function $u^{\infty}(\cdot, d)$ defined on $S^{1}$ is called the far-field of the scattered wave $u^{s}$ corresponding to incident direction $d$. We introduce a constant $\gamma_{2}:=\frac{e^{i \pi / 4}}{\sqrt{8 \pi \kappa}}$ and

$$
\Phi(x, y):=\frac{i}{4} H_{0}^{(1)}(\kappa|x-y|), \quad x \neq y, x, y \in R^{2},
$$

the fundamental solution to the Helmholtz equation in $R^{2}$, where $H_{0}^{(1)}$ is the Hankel function of the first kind of order zero. In this paper, we will consider the following

Inverse scattering problem for an obstacle with mixed boundary type: Given $u^{\infty}(\cdot, \cdot)$ on $S^{1} \times S^{1}$ for the scattering problem (2.1) - (2.4), reconstruct the shape of obstacle $D$, identify $\partial D_{I}$ and $\partial D_{D}$ and reconstruct the surface impedance $\sigma(x)$ on $\partial D_{I}$.

3. Presentation of the results. It is well known also, see [8], that the scattered field associated with the Herglotz incident field $v_{g}^{i}:=v_{g}$ defined by

$$
v_{g}(x):=\int_{S^{1}} e^{i \kappa x \cdot d} g(d) d s(d), \quad x \in R^{2}
$$

with $g \in L^{2}\left(S^{1}\right)$ is given by

$$
v_{g}^{s}(x):=\int_{S^{1}} u^{s}(x, d) g(d) d s(d), \quad x \in R^{2} \backslash D,
$$

and its far field is

$$
v_{g}^{\infty}(\hat{x}):=\int_{S^{1}} u^{\infty}(\hat{x}, d) g(d) d s(d), \quad \hat{x} \in S^{1} .
$$

We will need the following identity, see [8],

$$
u^{\infty}(\hat{x}, d)=-\gamma_{2} \int_{\partial D}\left\{\frac{\partial u^{s}(y, d)}{\partial \nu} e^{-i \kappa \hat{x} \cdot y}-\frac{\partial e^{-i \kappa \hat{x} \cdot y}}{\partial \nu} u^{s}(y, d)\right\} d s(y)
$$




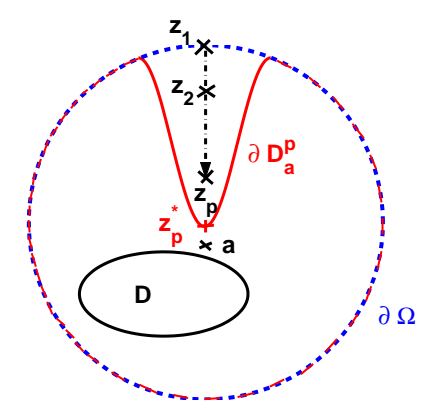

FIG. 3.1. Geometric configuration

and the representation formula for the scattered wave $\Phi^{s}(\cdot, z)$ in $R^{2} \backslash \bar{D}$ for point source $\Phi(\cdot, z)$

$$
\Phi^{s}(x, z)=-\int_{\partial D}\left\{\frac{\partial \Phi^{s}(y, z)}{\partial \nu(y)} \Phi(x, y)-\Phi^{s}(y, z) \frac{\partial \Phi(x, y)}{\partial \nu(y)}\right\} d s(y), \quad x, z \in R^{2} \backslash \bar{D}
$$

Assume that $\bar{D} \subset \subset \Omega$ for some known $\Omega$ with smooth boundary. For $a \in \Omega \backslash D$, denote by $\left\{z_{p}\right\} \subset \Omega \backslash \bar{D}$ a sequence tending to $a$. For any $z_{p}$, set $D_{a}^{p}$ a $C^{2}-$ regular domain such that $\bar{D} \subset D_{a}^{p}$ with $z_{q} \in \Omega \backslash \overline{D_{a}^{p}}$ for every $q=1,2, \cdots, p$ and that the Dirichlet interior problem on $D_{a}^{p}$ for the Helmholtz equation is uniquely solvable, see Figure 3.1 for the configuration. In this case, the Herglotz wave operator $\mathbb{H}$ defined from $L^{2}\left(S^{1}\right)$ to $L^{2}\left(\partial D_{a}^{p}\right)$ by

$$
\mathbb{H}[g](x):=v_{g}(x)=\int_{S^{1}} e^{i \kappa x \cdot d} g(d) d s(d)
$$

is injective, compact with dense range, see [8]. Let $z_{p}^{*}$ be a point on $\partial D_{a}^{p}$ near $z_{p}$ such that $z_{p}^{*} \rightarrow a$ as $z_{p} \rightarrow a$, as chosen in Figure 3.1. Denote by $\nu\left(z_{p}^{*}\right)$ the outward normal of $\partial D_{a}^{p}$ at $z_{p}^{*}$. Now we consider the sequence of point sources $\Phi\left(\cdot, z_{p}\right)$. For every $p$ fixed, we construct two density sequences $\left\{g_{n}^{p}\right\}$ and $\left\{f_{m}^{p}\right\}$ in $L^{2}\left(S^{1}\right)$ by the Tikhonov regularization such that

$$
\begin{gathered}
\left\|v_{g_{n}^{p}}-\Phi\left(\cdot, z_{p}\right)\right\|_{L^{2}\left(\partial D_{a}^{p}\right)} \rightarrow 0, \quad n \rightarrow \infty \\
\left\|v_{f_{m}^{p}}-\frac{\partial}{\partial \nu\left(z_{p}^{*}\right)} \Phi\left(\cdot, z_{p}\right)\right\|_{L^{2}\left(\partial D_{a}^{p}\right)} \rightarrow 0, \quad m \rightarrow \infty,
\end{gathered}
$$

where $\partial_{\nu\left(z_{p}^{*}\right)} \Phi\left(\cdot, z_{p}\right):=\nabla_{x} \Phi\left(x, z_{p}\right) \cdot \nu\left(z_{p}^{*}\right)$. Since both $v_{g_{n}^{p}}$ and $\Phi\left(\cdot, z_{p}\right)$ satisfy the same Helmholtz equation in $D_{a}^{p}$, (3.7) implies that

$$
\left\|v_{g_{n}^{p}}-\Phi\left(\cdot, z_{p}\right)\right\|_{H^{\frac{1}{2}}(\partial D)} \rightarrow 0, \quad n \rightarrow \infty
$$

and

$$
\left\|\frac{\partial}{\partial \nu} v_{g_{n}^{p}}-\frac{\partial}{\partial \nu} \Phi\left(\cdot, z_{p}\right)\right\|_{H^{-\frac{1}{2}}(\partial D)} \rightarrow 0, \quad n \rightarrow \infty
$$

Similarly, it follows from (3.8) that

$$
\left\|v_{f_{m}^{p}}-\frac{\partial}{\partial \nu\left(z_{p}^{*}\right)} \Phi\left(\cdot, z_{p}\right)\right\|_{H^{\frac{1}{2}(\partial D)}} \rightarrow 0, \quad m \rightarrow \infty
$$


and

$$
\left\|\frac{\partial}{\partial \nu} v_{f_{m}^{p}}-\frac{\partial}{\partial \nu}\left(\frac{\partial}{\partial \nu\left(z_{p}^{*}\right)} \Phi\left(\cdot, z_{p}\right)\right)\right\|_{H^{-\frac{1}{2}}(\partial D)} \rightarrow 0, \quad m \rightarrow \infty
$$

Multiplying (3.4) by $f_{m}^{p}(d) g_{n}^{p}(\hat{x})$ and integrating over $S^{1} \times S^{1}$, we have

$$
\begin{aligned}
& -\int_{S^{1}} \int_{S^{1}} u^{\infty}(-\hat{x}, d) f_{m}^{p}(d) g_{n}^{p}(\hat{x}) d s(\hat{x}) d s(d) \\
= & \gamma_{2} \int_{\partial D}\left\{\int_{S^{1}} \frac{\partial u^{s}(y, d)}{\partial \nu} f_{m}^{p}(d) d s(d) \cdot \int_{S^{1}} e^{i \kappa \hat{x} \cdot y} g_{n}^{p}(\hat{x}) d s(\hat{x})-\right. \\
& \left.\int_{S^{1}} \frac{\partial e^{i \kappa \hat{x} \cdot y}}{\partial \nu} g_{n}^{p}(\hat{x}) d s(\hat{x}) \cdot \int_{S^{1}} u^{s}(y, d) f_{m}^{p}(d) d s(d)\right\} d s(y) \\
= & \gamma_{2} \int_{\partial D}\left\{\frac{\partial v_{f_{m}^{p}}^{s}}{\partial \nu}(y) v_{g_{n}^{p}}^{i}(y)-\frac{\partial v_{g_{n}^{p}}^{i}}{\partial \nu}(y) v_{f_{m}^{p}}^{s}(y)\right\} d s(y) .
\end{aligned}
$$

From (3.9), (3.10) and (3.13), we have

$$
\begin{aligned}
& \lim _{n \rightarrow \infty} \int_{S^{1}} \int_{S^{1}} u^{\infty}(-\hat{x}, d) f_{m}^{p}(d) g_{n}^{p}(\hat{x}) d s(\hat{x}) d s(d) \\
= & \gamma_{2} \int_{\partial D}\left\{v_{f_{m}^{p}}^{s} \frac{\partial \Phi\left(y, z_{p}\right)}{\partial \nu(y)}-\frac{\partial v_{m}^{s}}{\partial \nu(y)} \Phi\left(y, z_{p}\right)\right\} d s(y) \\
= & \gamma_{2} v_{f_{m}^{p}}^{s}\left(z_{p}\right)
\end{aligned}
$$

from the Green formula, where $v_{f_{m}^{p}}^{s}(\cdot)$ is the scattered wave corresponding to incident wave $v_{f_{m}^{p}}^{i}(x)=\mathbb{H}\left[f_{m}^{p}\right](x)$.

Denote by $E^{s}\left(x, z_{p}\right)$ the scattered wave corresponding to the incident wave $\frac{\partial \Phi\left(x, z_{p}\right)}{\partial \nu\left(z_{p}^{*}\right)}$, which is well defined for every $x \in R^{2} \backslash \bar{D}$. Then it follows from (3.11), (3.12), the well posedness of the direct scattering problem and the use of interior estimate that

$$
E^{s}\left(x, z_{p}\right)=\lim _{m \rightarrow \infty} v_{f_{m}^{p}}^{s}(x), \quad x \in R^{2} \backslash \bar{D} .
$$

Finally, it follows from (3.14) that

$$
\lim _{m \rightarrow \infty} \lim _{n \rightarrow \infty} \int_{S^{1}} \int_{S^{1}} u^{\infty}(-\hat{x}, d) f_{m}^{p}(d) g_{n}^{p}(\hat{x}) d s(\hat{x}) d s(d)=\gamma_{2} E^{s}\left(z_{p}, z_{p}\right) .
$$

The reconstruction of $\partial D$ as well as its surface impedance in the coating part can be established based on (3.16). For this purpose, an analysis of $E^{s}(x, z)$ near $\partial D$ is the key point. We need the natural $C^{2}$ smoothness assumption on the regularity of $\partial D$. Precisely, for every point $a \in \partial D$, there exists a rigid transformation of coordinates under which the image of $a$ is $\mathbf{0}$ and a function $f \in C^{2}(-r, r)$ such that

$$
f(0)=\frac{d f}{d x}(0)=0, \quad D \cap B(\mathbf{0}, r)=\{(x, y) \in B(\mathbf{0}, r) ; y>f(x)\}
$$

in terms of the new coordinates where $B(\mathbf{0}, r)$ is the 2-dimensional ball of center $\mathbf{0}$ with radius $r$. 
For the points $a \in \partial D$, we choose the sequence $\left\{z_{p}\right\}_{p \in \mathbb{N}}$ included in $C_{a, \theta}$, where $C_{a, \theta}$ is a cone with center $a$, angle $\theta \in\left[0, \frac{\pi}{2}\right)$ and axis $\nu(a)$. The main theoretical result of this paper is as follows.

THEOREM 3.1. Assume that the boundary $\partial D$ is of class $C^{2}$ and $\sigma$ is a real valued Holder continuous function with positive lower bound. Then the boundary properties of the obstacle $D$ can be identified by the following indicator functions.

1. The obstacle boundary $\partial D$ can be constructed from the following property

$\lim _{p \rightarrow \infty} \lim _{m, n \rightarrow \infty}\left|\operatorname{Re}\left[\gamma_{2}^{-1} \int_{S^{1}} \int_{S^{1}} u^{\infty}(-\hat{x}, d) f_{m}^{p}(d) g_{n}^{p}(\hat{x}) d s(\hat{x}) d s(d)\right]\right|= \begin{cases}+\infty, & a \in \partial D, \\ <+\infty, & a \in \Omega \backslash \bar{D} .\end{cases}$

Precisely, we have the blowup rate:

$$
\lim _{m, n \rightarrow \infty} \operatorname{Re}\left[\gamma_{2}^{-1} \int_{S^{1}} \int_{S^{1}} u^{\infty}(-\hat{x}, d) f_{m}^{p}(d) g_{n}^{p}(\hat{x}) d s(\hat{x}) d s(d)\right]=\frac{ \pm 1}{4 \pi\left|\left(z_{p}-a\right) \cdot \nu(a)\right|}+
$$

$$
+O\left(|\ln | z_{p}-a||^{2}\right)
$$

where $z_{p}:=\left(z_{p, 1}, z_{p, 2}\right)$ and $a=\left(a_{1}, a_{2}\right)$. The sign $(+)$ is for $a \in \partial D_{D}$ while the sign (-) is for $a \in \partial D_{I}$.

2. The coating and the non-coating parts of $\partial D$ can also be distinguished from the following properties

$\lim _{p \rightarrow \infty} \lim _{m, n \rightarrow \infty} \frac{\operatorname{Im}\left[\gamma_{2}^{-1} \int_{S^{1}} \int_{S^{1}} u^{\infty}(-\hat{x}, d) f_{m}^{p}(d) g_{n}^{p}(\hat{x}) d s(\hat{x}) d s(d)\right]}{\left.|\ln |\left(z_{p}-a\right) \cdot \nu(a)\right|^{s}}= \begin{cases}+\infty, & a \in \partial D_{I}, \\ 0, & a \in \partial D_{D}\end{cases}$

by choosing any fixed $s \in(0,1)$.

3. The impedance coefficient on $\partial D_{I}$ can be detected by the following formula:

$\lim _{p \rightarrow \infty} \lim _{m, n \rightarrow \infty} \frac{\operatorname{Im}\left[\gamma_{2}^{-1} \int_{S^{1}} \int_{S^{1}} u^{\infty}(-\hat{x}, d) f_{m}^{p}(d) g_{n}^{p}(\hat{x}) d s(\hat{x}) d s(d)\right]}{|\ln |\left(z_{p}-a\right) \cdot \nu(a)||}=\frac{\kappa}{\pi} \sigma(a), \quad a \in \partial D_{I}$.

REMARK 3.2. The formula (3.18) is also true if $f_{m}^{p}$ is replaced by $g_{n}^{p}$. That is, the singularity of $\Phi\left(x, z_{p}\right)$ is theoretically enough for identifying $\partial D$. However, as the blowup rate in this 2 -dimensional case is of logarithmic order, it is not suitable to localize the obstacle clearly in the numerical experiments. Due to this reason, we introduced the density $f_{m}^{p}$ which is related to a stronger singularity $\frac{\partial}{\partial \nu\left(z_{p}^{*}\right)} \Phi\left(\cdot, z_{p}\right)$ to get a blowup rate of order $\left|z_{p}-a\right|^{-1}$. For the formulas (3.20) and (3.21), the stronger singularity of $\frac{\partial}{\partial \nu\left(z_{p}^{*}\right)} \Phi\left(\cdot, z_{p}\right)$ is necessary. Moreover, we can in fact use $\nu(a)$ instead of $\nu\left(z_{p}^{*}\right)$, since $\partial D$ has been determined in terms of (3.18). The formula (3.19) can also be used to distinguish the coated part $\partial D_{I}$ from the non-coated $\partial D_{I}$.

REMARK 3.3. Theorem 3.1 is stated for the case of single obstacle. But these results are still true for the multiple obstacle case with coated and non-coated parts.

REMARK 3.4. If $a \in D$, then the limit in (3.18) is conjectured to be $\infty$, see [11]. However up to now, we do not have the full answer. The approach in [15] can be used to justify it in the case where the frequency $\kappa$ is small enough. 
4. Proof of Theorem 3.1.. For any given point $a \in \partial D$, we firstly take the rotation $R_{a}$ and the translation $M_{a}$ such that

$$
R_{a}(\nu(a))=(0,1), \quad R_{a}(a)+M_{a}=\mathbf{0}
$$

in the new coordinate system $\tilde{x}$. Under the transform $\tilde{x}:=\mathbb{T}(x):=R_{a}(x)+M_{a}$, it follows that

$$
\mathbb{T}(\nu(a))=(0,1), \quad \mathbb{T}(a)=\mathbf{0}
$$

Define $\tilde{\sigma}(\tilde{x}):=\sigma(x)$ and consider the following two problems in the coordinate $\tilde{x}=\left(\tilde{x}_{1}, \tilde{x}_{2}\right)$ for any given $\tilde{z}=\left(\tilde{z}_{1}, \tilde{z}_{2}\right) \in R_{+}^{2}$. We set $\tilde{w}_{\tilde{\sigma}(\mathbf{0})}^{+}(\tilde{x}, \tilde{z})$ and $\tilde{w}_{D}^{+}(\tilde{x}, \tilde{z})$ to be two functions satisfying

$$
\begin{aligned}
& \left\{\begin{array}{l}
\Delta \tilde{w}_{\tilde{\sigma}(\mathbf{0})}^{+}=0, \quad \tilde{x} \in R_{+}^{2} \\
\left.\left(\frac{\partial}{\partial \tilde{x}_{2}} \tilde{w}_{\tilde{\sigma}(\mathbf{0})}^{+}+i \kappa \tilde{\sigma}(\mathbf{0}) \tilde{w}_{\tilde{\sigma}(\mathbf{0})}^{+}\right)(\tilde{x}, \tilde{z})\right|_{\tilde{x}_{2}=0}=-\left.\left(\frac{\partial}{\partial \tilde{x}_{2}}+i \kappa \tilde{\sigma}(\mathbf{0})\right) \frac{\partial}{\partial x_{2}} \Gamma(\tilde{x}, \tilde{z})\right|_{\tilde{x}_{2}=0},
\end{array}\right. \\
& \left\{\begin{array}{l}
\Delta \tilde{w}_{D}^{+}=0, \quad \tilde{x} \in R_{+}^{2}, \\
\left.\tilde{w}_{D}^{+}(\tilde{x}, \tilde{z})\right|_{\tilde{x}_{2}=0}=-\left.\frac{\partial}{\partial x_{2}} \Gamma(\tilde{x}, \tilde{z})\right|_{\tilde{x}_{2}=0}
\end{array}\right.
\end{aligned}
$$

respectively, where $\Gamma(\tilde{x}, \tilde{z})=\frac{1}{2 \pi} \ln \frac{1}{|\tilde{x}-\tilde{z}|}$ and the subscript $D$ in $\tilde{w}_{D}^{+}(\tilde{x}, \tilde{z})$ refers to the Dirichlet boundary condition in (4.2).

We give explicit solutions to these two problems in the following proposition.

Proposition 4.1. We have the explicit form of $w_{\tilde{\sigma}(\mathbf{0})}^{+}(\tilde{x}, \tilde{z})$

$$
\tilde{w}_{\tilde{\sigma}(\mathbf{0})}^{+}(\tilde{x}, \tilde{z})=\frac{1}{4 \pi} \int_{R} e^{i\left(\tilde{x}_{1}-\tilde{z}_{1}\right) \xi_{1}} e^{-\left(\tilde{x}_{2}+\tilde{z}_{2}\right)\left|\xi_{1}\right|} \frac{\left|\xi_{1}\right|+i \kappa \tilde{\sigma}(\mathbf{0})}{\left|\xi_{1}\right|-i \kappa \tilde{\sigma}(\mathbf{0})} d \xi_{1},
$$

while $\tilde{w}_{D}^{+}(\tilde{x}, \tilde{z})$ has the form

$$
\tilde{w}_{D}^{+}(\tilde{x}, \tilde{z})=-\frac{1}{4 \pi} \int_{R} e^{i\left(\tilde{x}_{1}-\tilde{z}_{1}\right) \xi_{1}} e^{-\left(\tilde{x}_{2}+\tilde{z}_{2}\right)\left|\xi_{1}\right|} d \xi_{1} .
$$

This proposition can be proven by expressing

$$
\tilde{w}_{\tilde{\sigma}(\mathbf{0})}^{+}(\tilde{x}, \tilde{z})=\left(U_{+}\left[\tilde{x}_{2}\right] \phi_{+}\right)\left(\tilde{x}_{1}\right), \quad \tilde{w}_{D}^{+}(\tilde{x}, \tilde{z})=\left(U_{+}\left[\tilde{x}_{2}\right] \phi_{-}\right)\left(\tilde{x}_{1}\right)
$$

in $R_{+}^{2}$ with $\left(U_{ \pm}\left[\tilde{x}_{2}\right] \phi\right)\left(\tilde{x}_{1}\right):=\frac{1}{2 \pi} \int_{R} e^{i \tilde{x}_{1} \xi_{1} \mp \tilde{x}_{2}\left|\xi_{1}\right|} \hat{\phi}\left(\xi_{1}, \tilde{z}\right) d \xi_{1}$ and computing the density functions $\phi_{ \pm}$from the boundary value problems (4.1), (4.2), where $\hat{\phi}$ is the 1-dimensional Fourier transform of $\phi$, see [19] for explicit computations.

Define

$$
w_{\sigma(a)}^{+}(x, z)=\tilde{w}_{\tilde{\sigma}(\mathbf{0})}^{+}(\mathbb{T} x, \mathbb{T} z), \quad w_{D}^{+}(x, z)=\tilde{w}_{D}^{+}(\mathbb{T} x, \mathbb{T} z)
$$

for $x, z \in R^{2} \backslash \bar{D}$ near $a$, which is well-defined by the definition of $\mathbb{T}$.

The next proposition gives the relation between $E^{s}(x, z)$ and $w_{\sigma(a)}^{+}(x, z), w_{D}^{+}(x, z)$ near the point $a$.

Proposition 4.2. If $a \in \partial D_{I}$, then there exist $\delta(a)>0$ and $C>0$ such that

$$
\left|\operatorname{Im} E^{s}(x, z)-\operatorname{Im} w_{\sigma(a)}^{+}(x, z)\right| \leq C, \text { for }(x, z) \in B_{+}(a, \delta(a)) \cap C_{a, \theta},
$$


$\left|\operatorname{Re} E^{s}(x, z)-\operatorname{Re} w_{\sigma(a)}^{+}(x, z)\right| \leq C|\ln | x-a|| \cdot|\ln | z_{p}-a||$, for $(x, z) \in B_{+}(a, \delta(a)) \cap C_{a, \theta}$,

where $B_{+}(a, \delta(a)):=B(a, \delta(a)) \cap\left(R^{2} \backslash D\right)$ and $B(a, \delta(a))$ is the ball of center $a$ and radius $\delta(a)$.

Similarly, if $a \in \partial D_{D}$, we obtain (4.5) and (4.6) by replacing $w_{\sigma(a)}^{+}$by $w_{D}^{+}$.

REMARK 4.3. The estimate of $\left|\operatorname{Re} E^{s}(x, z)-\operatorname{Re} w_{\sigma(a)}^{+}(x, z)\right|$ is not optimal. $W e$ do not need the term $|\ln | x-a||$. The upper bound in (4.6) can be replaced by $C_{\alpha}|z-a|^{-\alpha}$, for any $\alpha>0$ where $C_{\alpha}$ depends on $\alpha$. But to prove Theorem 3.1 the estimate given in (4.6) is enough. Now we can prove Theorem 3.1 based on these propositions.

Proof of Theorem 3.1.

Step A: It follows from Proposition 4.2 that

$$
\left|\operatorname{Re} E^{s}\left(x, z_{p}\right)-\operatorname{Re} w^{+}\left(x, z_{p}\right)\right| \leq C \ln \frac{1}{\left|z_{p}-a\right|}
$$

uniformly for all $x, z_{p}$ near any fixed point $a \in \partial D$, where $w^{+}\left(z_{p}, z_{p}\right)$ may be $w_{\sigma(a)}^{+}\left(z_{p}, z_{p}\right)$ or $w_{D}^{+}\left(z_{p}, z_{p}\right)$, depending on the position of $a$. For $w^{+}\left(z_{p}, z_{p}\right)=w_{D}^{+}\left(z_{p}, z_{p}\right)$, it follows from (4.4) that

$$
\operatorname{Re} w^{+}\left(z_{p}, z_{p}\right)=-\frac{1}{4 \pi} \int_{R} e^{-2\left|z_{p, 2}-a_{2}\right|\left|\xi_{1}\right|} d \xi_{1}=\frac{1}{4 \pi\left|z_{p, 2}-a_{2}\right|}
$$

while for $w^{+}\left(z_{p}, z_{p}\right)=w_{\sigma(a)}^{+}\left(z_{p}, z_{p}\right)$ it holds from (4.3) that

$$
\operatorname{Re} w^{+}\left(z_{p}, z_{p}\right)=\frac{1}{4 \pi} \int_{R} e^{-2\left|z_{p, 2}-a_{2}\right|\left|\xi_{1}\right|} \frac{\left|\xi_{1}\right|^{2}-\kappa^{2} \sigma^{2}(a)}{\left|\xi_{1}\right|^{2}+\kappa^{2} \sigma^{2}(a)} d \xi_{1}=-\frac{1}{4 \pi\left|z_{p, 2}-a_{2}\right|}+O(1)
$$

where $z_{p}=\left(z_{p, 1}, z_{p, 2}\right) \rightarrow a=\left(a_{1}, a_{2}\right) \in \partial D$ as $p \rightarrow \infty$. The application of the above relations in (4.7) leads to (3.19) and then $\lim _{p \rightarrow \infty}\left|\operatorname{Re} E^{s}\left(z_{p}, z_{p}\right)\right|=+\infty$. (3.18) is proven for $a \in \partial D$.

Suppose that $a$ is outside $\bar{D}$. We can construct $z_{p}^{*}, z_{p}$ tending to $a$ as we did for $a \in \partial D$. Recall that $E^{s}\left(x, z_{p}\right)$ satisfies

$$
\left\{\begin{array}{l}
\left(\Delta+\kappa^{2}\right) E^{s}\left(x, z_{p}\right)=0 \text { in } R^{2} \backslash \bar{D} \\
E^{s}\left(\cdot, z_{p}\right)=-\frac{\partial \Phi}{\partial \nu\left(z_{p}^{*}\right)}\left(x, z_{p}\right) \text { on } \partial D_{D} \\
\left(\frac{\partial}{\partial \nu}+i \kappa \sigma(x)\right) E^{s}\left(x, z_{p}\right)=-\left(\partial_{\nu}+i \kappa \sigma(x)\right) \frac{\partial \Phi}{\partial \nu\left(z_{p}^{*}\right)}\left(x, z_{p}\right) \text { on } \partial D_{I} \\
E^{s}\left(\cdot, z_{p}\right) \text { satisfies the Sommerfeld radiation conditions, }
\end{array}\right.
$$

where $\nu\left(z_{p}^{*}\right)$ is the unit outward normal on $\partial D_{a}^{p}$ at the point $z_{p}^{*}$. Hence the boundary condition is bounded with respect to $x$ in $H^{1 / 2}\left(\partial D_{D}\right)$ and $H^{-\frac{1}{2}}\left(\partial D_{I}\right)$, respectively, for $z_{p}^{*}, z_{p}$ near $a$. It follows from the well-posedness of the direct problem and interior estimates near $a$ (i.e. away from $\partial D$ ) that $E^{s}\left(x, z_{p}\right)$, and then $E^{s}\left(z_{p}, z_{p}\right)$, is bounded.

Step B. Let $a \in \partial D_{I}$. From (4.3) we have

$$
\tilde{w}_{\tilde{\sigma}(\mathbf{0})}^{+}(\tilde{z}, \tilde{z})=\frac{1}{4 \pi} \int_{R} e^{-2 \tilde{z}_{2}\left|\xi_{1}\right|} \frac{\left|\xi_{1}\right|+i \kappa \tilde{\sigma}(\mathbf{0})}{\left|\xi_{1}\right|-i \kappa \tilde{\sigma}(\mathbf{0})} d \xi_{1} .
$$

By taking the imaginary part and setting $\tilde{z}=\left(\tilde{z}_{1}, \tilde{z}_{2}\right)=R_{a}(z)+M_{a}$ for $z \in C(a, \theta)$, 
we get

$$
\begin{aligned}
\operatorname{Im}\left(4 \pi w_{\sigma(a)}^{+}(z, z)\right) & =4 \kappa \sigma(a) \int_{0}^{+\infty} \frac{e^{-2(z-a) \cdot \nu(a) r} r}{r^{2}+\kappa^{2} \sigma(a)^{2}} d r \\
& =4 \kappa \sigma(a)[-\ln (\kappa \sigma(a))-\ln ((z-a) \cdot \nu(a))+ \\
& \left.\quad 2 \int_{0}^{+\infty} \ln \left(r^{2}+\kappa^{2}|(z-a) \cdot \nu(a)|^{2} \sigma^{2}(a)\right) e^{-2 r} d r\right]
\end{aligned}
$$

which leads to the first relation in (3.20) by dividing by $|\ln ((z-a) \cdot \nu(a))|^{s}$ for $0<(z-a) \cdot \nu(a)<1$ with $0<s<1$ using (4.5) and (3.16). The representation (3.21) for $\sigma(a)$ can be gotten from the above relation by dividing by $|\ln ((z-a) \cdot \nu(a))|$ for $0<(z-a) \cdot \nu(a)<1$.

Step C. Let $a \in \partial D_{D}$. Proposition 4.2 for $w_{D}^{+}(x, z)$ and (3.14) imply the second relation in $(3.20)$, noticing the fact that $\operatorname{Im} w_{D}^{+}(z, z)=0$.

The rest of this section is devoted to the proof of Proposition 4.2. As we said in the introduction, in the $2 \mathrm{D}$ case, we need more singularity analysis than in [19]. This is due to the use of the more singular point source $\frac{\partial}{\partial \nu\left(z_{p}^{*}\right)} \Phi\left(\cdot, z_{p}\right)$. We give the detailed analysis and refer to [19] for the steps which do not need important changes.

4.1. Proof of Proposition 4.2.. We give the proof for $a \in \partial D_{I}$. The proof for $a \in \partial D_{D}$ is similar.

Let $\tilde{E}^{s}\left(x, z_{p}\right)$ be the solution of

$$
\left\{\begin{array}{l}
\left(\Delta+\kappa^{2}\right) \tilde{E}^{s}\left(x, z_{p}\right)=0 \text { in } R^{2} \backslash \bar{D} \\
\left(\frac{\partial}{\partial \nu}+i \kappa \sigma(x)\right) \tilde{E}^{s}\left(x, z_{p}\right)=-\left(\partial_{\nu}+i \sigma(x)\right) \frac{\partial}{\partial \nu\left(z_{p}^{*}\right)} \Phi\left(x, z_{p}\right) \text { on } \partial D \\
\tilde{E}^{s}(\cdot, z) \text { satisfies the Sommerfeld radiation condition. }
\end{array}\right.
$$

Hence $\left(E^{s}-\tilde{E}^{s}\right)\left(x, z_{p}\right)$ satisfies

$$
\left\{\begin{array}{l}
\left(\Delta+\kappa^{2}\right)\left(E^{s}-\tilde{E}^{s}\right)\left(x, z_{p}\right)=0 \text { in } R^{2} \backslash \bar{D} \\
\left(\frac{\partial}{\partial \nu}+i \kappa \sigma(x)\right)\left(E^{s}-\tilde{E}^{s}\right)\left(x, z_{p}\right)=0 \text { on } \partial D_{I} \\
\left(E^{s}-\tilde{E}^{s}\right)\left(\cdot, z_{p}\right)=-\frac{\partial}{\partial \nu\left(z_{p}^{*}\right)} \Phi\left(x, z_{p}\right)-\tilde{E}^{s} \text { on } \partial D_{D} \\
\left(E^{s}-\tilde{E}^{s}\right)(\cdot, z) \text { satisfies the Sommerfeld radiation condition. }
\end{array}\right.
$$

We state $H_{\sigma}(x, z):=\tilde{E}(x, z)+\partial_{\nu\left(z_{p}^{*}\right)} \Phi(x, z)$. Hence $H$ satisfies

$$
\left\{\begin{array}{l}
\left(\Delta+\kappa^{2}\right) H_{\sigma}(x, z)=-\nabla \delta(x, z) \cdot \nu\left(z_{p}^{*}\right) \text { in } R^{2} \backslash \bar{D} \\
\left(\frac{\partial}{\partial \nu}+i \kappa \sigma(x)\right) H_{\sigma}(x, z)=0 \text { on } \partial D \\
H_{\sigma}(\cdot, z) \text { satisfies the Sommerfeld radiation condition. }
\end{array}\right.
$$

We have the following estimates:

$$
\left\{\begin{array}{l}
\left|G_{\sigma}(x, z)\right| \leq c|\ln | x-z|| \\
\left|\nabla G_{\sigma}(x, z)\right| \leq c|x-z|^{-1} \quad \text { in } R^{2} \backslash D \text { where } c \text { is a positive constant. } \\
\left|H_{\sigma}(x, z)\right| \leq c|x-z|^{-1} \\
\left|\nabla H_{\sigma}(x, z)\right| \leq c|x-z|^{-2}
\end{array}\right.
$$

The justification of these properties can be derived following, for instance, the approach of [24] and [25] since an explicit form of a local fundamental solution for the 
half space case can be derived as we did in Proposition4.1. See also [18] and [12] for the case of elliptic problems with rough coefficients.

From these estimates, we deduce that $\tilde{E}\left(\cdot, z_{p}\right)$ and its derivatives are bounded for $x \in \partial D_{D}$ and $z_{p}$ near $a \in \partial D_{I}$. The well posed-ness of (4.11) implies that $\left(E^{s}-\tilde{E}^{s}\right)\left(\cdot, z_{p}\right)$ is bounded in $H_{l o c}^{1}\left(R^{2} \backslash \bar{D}\right)$ for $z_{p}$ near $a$. Introducing a cutoff function near the point $a$ and using (4.11), we deduce that $\left(E^{s}-\tilde{E}^{s}\right)\left(\cdot, z_{p}\right)$ is bounded for $x$ near $\partial D_{I}$ and $z_{p}$ near $a$.

This means that we can replace in Proposition $4.2 E^{s}$ by $\tilde{E}^{s}$.

We introduce $w_{\sigma}^{s}\left(\cdot, z_{p}\right)$ as the solution of

$$
\left\{\begin{array}{l}
\left(\Delta+\kappa^{2}\right) w_{\sigma}^{s}\left(x, z_{p}\right)=0 \text { in } R^{2} \backslash \bar{D} \\
\left(\frac{\partial}{\partial \nu}+i \kappa \sigma(x)\right) w_{\sigma}^{s}\left(x, z_{p}\right)=-\left(\partial_{\nu}+i \sigma(x)\right) \frac{\partial}{\partial \nu(a)} \Phi\left(\cdot, z_{p}\right) \text { on } \partial D \\
w_{\sigma}^{s}(\cdot, z) \text { satisfies the Sommerfeld radiation condition, }
\end{array}\right.
$$

and denote by $w_{\sigma(a)}^{s}\left(\cdot, z_{p}\right)$ the solution of (4.14) replacing $\sigma(x)$ by $\sigma(a)$. Then we have

LEMma 4.4. There exist $\delta(a)>0$ and $C(R)>0$ such that

$$
\begin{gathered}
\left|\left(\tilde{E}^{s}-w_{\sigma}^{s}\right)\left(x, z_{p}\right)\right| \leq C(R)|\ln d(x, \partial D)| \cdot\left|\ln d\left(z_{p}, \partial D\right)\right|, \\
\left|\operatorname{Im}\left(\tilde{E}^{s}-w_{\sigma}^{s}\right)\left(x, z_{p}\right)\right| \leq C(R), \quad\left|\left(w_{\sigma}^{s}-w_{\sigma(a)}^{s}\right)\left(x, z_{p}\right)\right| \leq C(R)
\end{gathered}
$$

for $z_{p} \in B(a, \delta(a)) \cap C_{a, \theta}$ and $x \in\left(R^{2} \backslash D\right) \cap B(0, R)$, for any $R>0$ fixed.

Let $w_{\sigma(a), \Phi}^{s}(\cdot, z)$ be the solution of

$$
\left\{\begin{array}{l}
\left(\Delta+\kappa^{2}\right) w_{\sigma(a), \Phi}^{s}(x, z)=0 \text { in } \Omega \backslash \bar{D}, \\
\left(\frac{\partial}{\partial \nu}+i \kappa \sigma(a)\right) w_{\sigma(a) \Phi}^{s}(x, z)=-\left(\frac{\partial}{\partial \nu}+i \kappa \sigma(a)\right) \frac{\partial}{\partial \nu(a)} \Phi\left(x, z_{p}\right) \text { on } \partial D \\
w_{\sigma(a), \Phi}^{s}(\cdot, z)=-\frac{\partial}{\partial \nu(a)} \Phi\left(x, z_{p}\right) \text { on } \partial \Omega
\end{array}\right.
$$

and $w_{\sigma(a), \Gamma}^{s}(\cdot, z)$ be the solution of (4.15) replacing $\Phi$ by $\Gamma$. Then we have

LEMMA 4.5. There exists $C>0$ such that

$$
\left|\left(w_{\sigma(a)}^{s}-w_{\sigma(a), \Phi}^{s}\right)(x, z)\right| \leq C, \quad\left|\left(w_{\sigma(a), \Phi}^{s}-w_{\sigma(a), \Gamma}^{s}\right)(x, z)\right| \leq C
$$

for $z \in \Omega \backslash D$ near $D$ and $x \in \Omega \backslash D$.

We define $w_{\sigma(a)}^{s, 0}$ to be the solution of (4.15) replacing $\Phi$ by $\Gamma$ and the Helmholtz equation by the Laplace equation. Then we have

Lemma 4.6. There exists $C>0$ such that $\left|\left(w_{\sigma(a), \Gamma}^{s}-w_{\sigma(a)}^{s, 0}\right)(x, z)\right| \leq C$, for $z \in \Omega \backslash D$ near $D$ and $x \in \Omega \backslash D$. Finally, we have

LEMma 4.7. There exist $C>0, \delta(a)>0$ such that

1. $\left|\left(\operatorname{Im} w_{\sigma(a)}^{s, 0}-\operatorname{Im} w_{\sigma(a)}^{+}\right)(x, z)\right| \leq C$ for $(x, z) \in B(a, \delta(a)) \cap C_{a, \theta}$.

2. $\left|\left(\operatorname{Re} w_{\sigma(a)}^{s, 0}-\operatorname{Re} w_{\sigma(a)}^{+}\right)(x, z)\right| \leq C\left|\ln d\left(z_{p}, \partial D\right)\right|$ for $(x, z) \in B(a, \delta(a)) \cap C_{a, \theta}$. By combining all the lemmas stated above, we end the proof of Proposition 4.2.

In the proofs of these lemmas we do not, in general, specify the interdependency of the constants appearing in the estimates. However we distinguish the constant depending on the angle $\theta$ and the ones which do not depend.

Proof of Lemma 4.4. The function $\tilde{E}^{s}\left(x, z_{p}\right)-w_{\sigma}^{s}\left(x, z_{p}\right)$ satisfies

$$
\left\{\begin{array}{l}
\left(\Delta+\kappa^{2}\right)\left(\tilde{E}^{s}-w_{\sigma}^{s}\right)\left(x, z_{p}\right)=0 \text { in } R^{2} \backslash \bar{D}, \\
\left(\frac{\partial}{\partial \nu}+i \kappa \sigma\right)\left(\tilde{E}^{s}-w_{\sigma}^{s}\right)\left(x, z_{p}\right)=-\left(\frac{\partial}{\partial \nu}+i \kappa \sigma\right) \nabla \Phi \cdot\left[\nu\left(z_{p}^{*}\right)-\nu(a)\right] \quad \text { on } \quad \partial D, \\
\left(\tilde{E}^{s}-w_{\sigma}^{s}\right)\left(\cdot, z_{p}\right) \text { satisfies the Sommerfeld radiation condition. }
\end{array}\right.
$$


We need the following lemma.

LEMMA 4.8. We have the estimate:

$$
\left|\nu\left(z_{p}^{*}\right)-\nu(a)\right| \leq C\left|z_{p}^{*}-a\right|,
$$

for $z_{p}^{*}$ near a where $C$ is a positive constant. Proof of Claim 4.8. Take a point $b \in \partial \Omega$ and connect it to $a$ by a $C^{3}$ smooth curve $l$ such that $l(0)=a, l(1)=b, l(s) \in \Omega \backslash \bar{D}$ $(s \in(0,1))$. By Theorem 7.1 of [11], there is a $C^{2}$ strict deformation family $\left\{D_{a}^{l(s)}\right\}$ of $a$ and $\partial \Omega$. That is, each $\partial D_{a}^{l(s)}$ is $C^{2}$ diffeomorphic to the unit circle and $\left\{D_{a}^{l(s)}\right\}$ satisfies the following properties:

(i) $a \in \partial D_{a}^{l(0)}, D \subset D_{a}^{l(0)} \subset \Omega$.

(ii) $\partial D_{a}^{l(1)}=\partial \Omega, \overline{D_{a}^{l(s)}} \subset D_{a}^{l\left(s^{\prime}\right)}\left(0 \leq s<s^{\prime} \leq 1\right)$.

(iii) $l$ intersects $\partial D_{a}^{l(s)}$ at $l(s)$.

(iv) $\partial D_{a}^{l(s)}$ depends $C^{2}$ smoothly on $s \in[0,1]$.

For every $s \in[0,1]$ we denote $\nu(l(s))$ to be the unit normal of $\partial D_{a}^{l(s)}$ at $l(s)$. From (iv), the map $s \in[0,1] \rightarrow \nu(l(s))$ is $C^{1}$. Choosing $l$ to be a one to one curve near $a$, we deduce that the map $l(s) \in l([0,1]) \rightarrow \nu(l(s))$ is also $C^{1}$ near $l(0)$. Now let $\left\{s_{p}\right\} \subset[0,1]$ be such that $s_{p} \rightarrow 0(p \rightarrow \infty)$ and $l$ be a $C^{3}$ smooth curve such that $z_{p}^{*}:=l\left(s_{p}\right)$ and $\left\{z_{p}\right\}_{p \in \mathbb{N}} \subset l([0,1])$. Since for every $p, z_{p}$ and $z_{p}^{*}$ are in $\Omega \backslash \bar{D}$, then we can always choose $l$ such that $l(s) \in \Omega \backslash \bar{D},(s \in[0,1])$. We set $D_{a}^{p}:=D_{a}^{l\left(s_{p}\right)}$. Hence the sequence $\nu\left(z_{p}^{*}\right)$ satisfies Claim 4.8 .

The function $\operatorname{Im}\left(\tilde{E}^{s}-w_{\sigma}^{s}\right)$ satisfies

$$
\left\{\begin{array}{l}
\left(\Delta+\kappa^{2}\right) \operatorname{Im}\left(\tilde{E}^{s}-w_{\sigma}^{s}\right)(x, z)=0 \text { in } R^{2} \backslash \bar{D} \\
\quad \frac{\partial}{\partial \nu} \operatorname{Im}\left(\tilde{E}^{-} w_{\sigma}^{s}\right)(x, z)=\left[\kappa \sigma \operatorname{Re}\left(\tilde{E}^{s}-w_{\sigma}^{s}\right)-\left[\frac{\partial}{\partial \nu} \operatorname{Im} \nabla \Phi+\kappa \sigma \operatorname{Re} \nabla \Phi\right] \cdot\left[\nu\left(z_{p}^{*}\right)-\nu(a)\right]\right](x, z) \\
\quad \text { on } \partial D
\end{array}\right.
$$

Hence we have

$$
\begin{gathered}
-\operatorname{Im}\left(\tilde{E}^{s}-w_{\sigma}^{s}\right)\left(x, z_{p}\right)=\int_{\partial D} F\left(y, z_{p}\right) G_{N}(y, x) d s(y)+ \\
+\int_{\partial B_{R}} \operatorname{Im}\left(\tilde{E}^{s}-w_{\sigma}^{s}\right)\left(y, z_{p}\right) \frac{\partial}{\partial \nu} G_{N}(y, x) d s(y)
\end{gathered}
$$

where

$F\left(x, z_{p}\right):=\kappa \sigma(x) \operatorname{Re}\left(\tilde{E}^{s}-w_{\sigma}^{s}\right)(x, z)+\left[-\frac{\partial}{\partial \nu} \operatorname{Im} \nabla \Phi-\kappa \sigma(x) \operatorname{Re} \nabla \Phi\right](x, z) \cdot\left[\nu\left(z_{p}^{*}\right)-\nu(a)\right]$

and $G_{N}(x, y)$ is the Green's function of the problem given by the Helmholtz equation in $B_{R} \backslash \bar{D}$ with Neumann boundary condition on $\partial D$ and Dirichlet boundary condition on $\partial B_{R}$. The normal $\nu$ is oriented outside $B \backslash \bar{D}$.

From (4.16), we have

$$
-\left(\tilde{E}^{s}-w_{\sigma}^{s}\right)\left(x, z_{p}\right)=\int_{\partial D} G_{\sigma}(x, y)\left(\frac{\partial}{\partial \nu}+i \kappa \sigma(y)\right)\left[\nabla \Phi \cdot\left(\nu\left(z_{p}^{*}\right)-\nu(a)\right)\right]\left(y, z_{p}\right) d s(y) .
$$

Hence

$$
\left|\left(\tilde{E}^{s}-w_{\sigma}^{s}\right)\left(x, z_{p}\right)\right| \leq c \int_{\partial D}(\ln |x-y|)\left|y-z_{p}\right|^{-2}\left|z_{p}^{*}-a\right| d s(y)
$$


For $y \in \partial D$ and $z_{p} \in C_{a, \theta}$ we have the inequality $\left|z_{p}-a\right| \leq C(\theta)\left|z_{p}-y\right|$. Applying this inequality to $z_{p}^{*}$, enlarging $\theta$ if necessary, we have

$$
\left|\left(\tilde{E}^{s}-w_{\sigma}^{s}\right)\left(x, z_{p}\right)\right| \leq c \int_{\partial D}(\ln |x-y|)\left|y-z_{p}\right|^{-1} d s(y)
$$

since $\left|z_{p}^{*}-y\right| \leq\left|z_{p}-y\right|$, for $y \in \partial D$ and $z_{p}$ near $a$. Hence for every $\alpha>0$ there exists $C_{\alpha}>0$ such that

$$
\left|\left(\tilde{E}^{s}-w_{\sigma}^{s}\right)\left(x, z_{p}\right)\right| \leq C_{\alpha}\left|x-z_{p}\right|^{-\alpha}
$$

From the explicit form of $\Phi$, we have $\left|\frac{\partial}{\partial \nu} \operatorname{Im} \nabla \Phi\left(x, z_{p}\right)\right| \cdot\left|\nu\left(z_{p}^{*}\right)-\nu(a)\right| \leq c\left|x-z_{p}\right|^{-1} \mid z_{p}^{*}-$ $a \mid$ and $\operatorname{Re} \nabla \Phi\left(x, z_{p}\right)|\cdot| \nu\left(z_{p}^{*}\right)-\nu(a)|\leq c| x-\left.z_{p}\right|^{-1}\left|z_{p}^{*}-a\right|$. Hence $F\left(y, z_{p}\right) \leq C_{\alpha}\left|y-z_{p}\right|^{-\alpha}$ for $y \in \partial D$. Using the estimate $\left|G_{N}(x, y)\right| \leq C_{\alpha} \ln |x-y|$ and choosing $\alpha<1$ we deduce from (4.18) that

$$
\left|\operatorname{Im}\left(\tilde{E}^{s}-w_{\sigma}^{s}\right)\left(x, z_{p}\right)\right| \leq C_{\alpha}
$$

We have the first estimate of Lemma 4.4 from (4.19), i.e

$$
\left|\left(\tilde{E}^{s}-w_{\sigma}^{s}\right)\left(x, z_{p}\right)\right| \leq C \ln d(x, \partial D)|\cdot| \ln d\left(z_{p}, \partial D\right) \mid .
$$

Now consider the third estimate of Lemma 4.4. We set $R(x, z):=w_{\sigma}^{s}(x, z)-w_{\sigma(a)}^{s}(x, z)$. Then it satisfies

$$
\left\{\begin{array}{l}
\left(\Delta+\kappa^{2}\right) R(x, z)=0 \text { in } R^{2} \backslash \bar{D} \\
\frac{\partial R(x, z)}{\partial \nu}+i \kappa \sigma(a) R(x, z)=-i \kappa(\sigma(x)-\sigma(a))\left(w_{\lambda}^{s}(x, z)+\frac{\partial}{\partial \nu(a)} \Phi(x, z)\right) \quad \text { on } \quad \partial D, \\
R(\cdot, z) \text { satisfies the Sommerfeld radiation condition. }
\end{array}\right.
$$

From (4.20), we have the representation:

$$
R(x, z)=-\int_{\partial D} i \kappa(\sigma(y)-\sigma(a)) G_{\sigma(a)}(y, x)\left(w_{\sigma}^{s}+\frac{\partial}{\partial \nu(a)} \Phi\right)(y, z) d s(y) \text {, for }(x, z) \in R^{2} \backslash \bar{D} .
$$

We define $K(x, z):=-\left(\frac{\partial}{\partial \nu}+i \kappa \sigma(x)\right) \frac{\partial}{\partial \nu(a)} \Phi(x, z)$. From (4.14) we have the following representation

$$
w_{\sigma}^{s}(x, z)=\int_{\partial D} G_{\sigma(a)}(y, x) K(y, z) d s(y),
$$

hence due to the estimates of the Green's function $G_{\sigma(a)}(x, y)$ and $\Phi(x, y)$, we have

$$
\left|w_{\sigma}^{s}(x, z)\right| \leq \int_{\partial D}|\ln (|y-x|)||z-y|^{-2} d s(y) \leq \frac{c}{|x-z|} .
$$

From (4.21) and the Holder regularity of $\sigma(x)$, we deduce that

$$
|R(x, z)| \leq c \int_{\partial D}|y-a|^{\beta} \ln (|y-x|)|| z-\left.y\right|^{-1} d s(y) .
$$

From the inequality $|y-a| \leq c(\theta)|y-z|$ for $y \in \partial D$ and $z \in C_{a, \theta} \cap B(a, \delta(a))$ we have

$$
\frac{|y-a|^{\beta}}{|y-z|} \leq \frac{c(\theta)^{\beta} C}{|y-z|^{1-\beta}}
$$


which implies

$$
|R(x, z)| \leq \int_{\partial D} \frac{c(\theta)^{\beta} C|\ln | y-x||}{|y-z|^{1-\beta}} d y
$$

and therefore $|R(x, z)|=O(1)$ for $x \in R^{2} \backslash D$ and $z \in C_{a, \theta} \cap B(0, R)$.

Proofs of Lemma 4.5 and Lemma 4.6.

Similarly to the proof of Lemma 4.4, the proofs are based on the use of integral representations and the pointwise estimates of the Green's functions of the corresponding problems. So, we omit the details.

Proof of Lemma 4.7. Since $w_{\sigma(a)}^{s, 0}$ satisfies

$$
\left\{\begin{array}{l}
\Delta w_{\sigma(a)}^{s, 0}(x, z)=0 \text { in } \Omega \backslash \bar{D}, \\
\left(\frac{\partial}{\partial \nu}+i \kappa \sigma(a)\right)\left(w_{\sigma(a)}^{s, 0}(\cdot, z)\right)=-\left(\frac{\partial}{\partial \nu}+i \kappa \sigma(a)\right) \frac{\partial}{\partial \nu(a)} \Gamma \quad \text { on } \quad \partial D, \\
w_{\sigma(a)}^{s, 0}(\cdot, z)=-\frac{\partial}{\partial \nu(a)}(\Gamma) \text { on } \partial \Omega,
\end{array}\right.
$$

then it is clear that $G_{\sigma(a)}^{0}:=w_{\sigma(a)}^{s, 0}(x, y)+\partial_{\nu(a)} \Gamma(x, y)$ satisfies

$$
\left\{\begin{array}{l}
\Delta\left(G_{\sigma(a)}^{0}\right)(x, z)=-\frac{\partial}{\partial \nu(a)} \delta(x-y) \text { in } \Omega \backslash \bar{D} \\
\left(\frac{\partial}{\partial \nu}+i \kappa \sigma(a)\right)\left(G_{\sigma(a)}^{0}\right)(\cdot, z)=0 \quad \text { on } \partial D \\
\left(G_{\sigma(a)}^{0}\right)(\cdot, z)=0 \text { on } \partial \Omega
\end{array}\right.
$$

We can assume without loss of generality that $a=(0,0)$ and $\nu(a)=(0,1)$ by using the rigid transformation of coordinates $\left[R_{a}(\nu(a))+M_{a}\right]$. Let $\xi=F(x)$ be the local change of variables

$$
\xi_{1}=x_{1}, \quad \xi_{2}=x_{2}-f\left(x_{1}\right),
$$

where $f$ is the function defined in the introduction. We have the following properties:

$$
\left\{\begin{array}{l}
c_{1}|x-z| \leq|F(x)-F(z)| \leq c_{2}|x-z|, \\
|F(x)-x| \leq c_{3}|x|^{2} \\
|D F(x)-I| \leq c_{4}|x|
\end{array}\right.
$$

for $x, z$ near the point $a$, where $c_{i}(i=1,2,3,4)$ are positive constants, which is due to hypothesis on the regularity of $\partial D$.

Let $x, z$ be points near $a$. From (4.23), we deduce that $\tilde{G}_{\sigma(a)}^{0}(\xi, \eta)=G_{\sigma(a)}^{0}(x, z)$ satisfies:

$$
\left\{\begin{array}{l}
\nabla_{\xi} \cdot B(\xi) \nabla_{\xi} \tilde{G}_{\sigma(a)}^{0}=-J^{T}(\xi) \nabla_{\xi} \delta(\xi-\eta) \cdot(0,1) \text { near } F(a), \\
\left|J^{-T} \nu\right| B(\xi) \nabla_{\xi} \tilde{G}_{\sigma(a)}^{0} \cdot \tilde{\nu}+i \kappa \sigma(a) \tilde{G}_{\sigma(a)}^{0}=0 \text { on } \partial R_{+}^{2} \text { near } F(a)
\end{array}\right.
$$

where $\xi:=F(x)$ and $\eta:=F(z), B:=J J^{T}$ and $J:=\frac{\partial \xi}{\partial x}\left(F^{-1}(\xi)\right)$ and $\tilde{\nu}:=(0,1)$ is the unit normal to $\partial R_{+}^{2}$. We denoted by $J^{-T}$ the adjoint of $J^{-1}$. We have from (4.25) that

$$
\left|J^{T}(\xi)-J^{T}(0)\right| \leq c|\xi|, \quad|B(\xi)-B(0)| \leq c|\xi|
$$

and $J(0)=B(0)=I$. 
We set $\Gamma_{\sigma(a)}(x, z):=\left(w_{\sigma(a)}^{+}+\frac{\partial}{\partial x_{2}} \Gamma\right)(x, z)$ and write $\tilde{R}(\xi, \eta):=\tilde{G}_{\sigma(a)}^{0}(\xi, \eta)-$ $\Gamma_{\sigma(a)}(\xi, \eta)$. Then the function $\tilde{R}(\cdot, \eta)$ satisfies

$$
\left\{\begin{array}{l}
\nabla_{\xi} \cdot B(\xi) \nabla_{\xi} \tilde{R}=\nabla_{\xi} \cdot(I-B) \nabla_{\xi} \Gamma_{\sigma(a)}+\left(I-J^{T}(\xi)\right) \nabla_{\xi} \delta(\xi-\eta) \cdot(0,1), \\
B(\xi) \nabla_{\xi} \tilde{R} \cdot \tilde{\nu}+i \kappa \sigma(a) \tilde{R}=(I-B) \nabla_{\xi} \Gamma_{\sigma(a)} \cdot \tilde{\nu}+i \kappa \sigma(a)\left(1-\left|J^{-T} \nu\right|^{-1}\right) \tilde{R}
\end{array}\right.
$$

where the first relation holds in $R_{+}^{2}$ near $F(a)$, while the second one is satisfied on $\partial R_{+}^{2}$ near $F(a)$.

We remark that $\left(I-J^{T}(\xi)\right)$ is equal to the matrix given by the two line vectors $(0,-f(\xi))$ and $(0,0)$. Hence we have

$$
\left(I-J^{T}(\xi)\right) \nabla_{\xi} \operatorname{Im} \Gamma_{\sigma(a)}(\xi, \eta) \cdot(0,1)=0 .
$$

With this remark, the problem (4.27) is exactly the one studied in [19]. We write $\partial B_{r}^{+}=S_{r} \cup S_{r}^{c}$ with $S_{r}:=\partial B_{r}^{+} \cap \partial F(D)$. Arguing as in [19], we obtain the following estimate

$$
|\operatorname{Im} \tilde{R}(\xi, \eta)|<c \text { for } \xi \in S_{r} \text { and } \eta \in C_{F(a), \theta} \text {. }
$$

and

$|\operatorname{Re} \tilde{R}(\xi, \eta)|<c|\ln | \xi-\eta||$ for $\xi \in S_{r}$ and $\eta \in C_{F(a), \theta}$.

We go back to $R(x, z):=G_{\sigma(a)}^{0}(x, z)-\Gamma_{\sigma(a)}(x, z)$. We have

$$
R(x, z)=G_{\sigma(a)}^{0}(x, z)-\Gamma_{\sigma(a)}(F(x), F(z))+\Gamma_{\sigma(a)}(F(x), F(z))-\Gamma_{\sigma(a)}(x, z),
$$

which can be rewritten as

$$
R(x, z)=\tilde{R}(F(x), F(z))+\left[\Gamma_{\sigma(a)}(F(x), F(z))-\Gamma_{\sigma(a)}(F(x), z)\right]+
$$

$+$

$$
\left[\Gamma_{\sigma(a)}(F(x), z)-\Gamma_{\sigma(a)}(x, z)\right]
$$

By the same argument as in [19], we end up with the estimate:

$$
|\operatorname{Im} R(x, z)| \leq c(\theta)
$$

for $x \in B(a, \delta(a))$ such that $F(x) \in S_{r}$ and $z \in C_{a, \theta} \cap B(a, \delta(a))$.

For $z \in C_{a, \theta} \cap B\left(a, \frac{\delta(a)}{2}\right)$ and $x \in[\partial B(a, \delta(a))] \cap R^{2} \backslash \bar{D}$, we have

$$
|\operatorname{Im} R(x, z)| \leq c
$$

with some positive constant $c$, because $C_{a, \theta} \cap B\left(a, \frac{\delta(a)}{2}\right)$ and $[\partial B(a, \delta(a))] \cap R^{2} \backslash \bar{D}$ are separated sets. Since in $B(a, \delta(a)) \cap\left(R^{2} \backslash \bar{D}\right)$, we have $\Delta_{x} \operatorname{Im} R(x, z)=0$, then using (4.29) and (4.30) we have $|\operatorname{Im} R(x, z)| \leq c(\theta)$ for $x \in\left[R^{2} \backslash D\right] \cap B(a, \delta(a))$ and $z \in C_{a, \theta} \cap B\left(a, \frac{\delta(a)}{2}\right)$, by the maximum principle.

Similarly we have $|\operatorname{Re} R(x, z)| \leq C|\ln | x-z||$ for $x \in B(a, \delta(a))$ such that $F(x) \in$ $S_{r}$ and $z \in C_{a, \theta} \cap B(a, \delta(a))$. Hence $|\operatorname{Re} R(x, z)| \leq C|\ln d(z, \partial D)|$ for $x \in B(a, \delta(a))$ such that $F(x) \in S_{r}$ and $z \in C_{a, \theta} \cap B(a, \delta(a))$ which is the counterpart of (4.30) for $\operatorname{Re} \tilde{R}$. The rest of the proof is the same as the one for the imaginary part. 
5. Numerical tests. In this section, we consider two reconstruction model problems for numerical tests based on Theorem 3.1. In the first model we take the obstacle to be a disc and on its whole boundary we impose the impedance boundary condition. The purpose of considering such a model is to show the influence of wave number $\kappa$ and singularity strength on the inversion scheme. In the second model, we consider a non-convex obstacle with mixed boundary conditions. We check our inversion formulas fully, especially for the identification of different type of boundary. Also we show the effect of the non-convex part on the inversion performance.

In the reconstruction scheme, the approximation of $\Phi\left(x, z_{p}\right)$ and $\Phi_{\nu(a)}\left(x, z_{p}\right)$ by the Herglotz wave function plays a key role. To do this, we need to construct the approximate domain $D_{a}^{p}$ in the way mentioned in Section 2. Then their density functions can be determined by the standard argument of minimum norm solution of the integral equation of the first kind. In this way the numerators in (3.18), (3.20) and (3.21) can be computed for every sequence of points $\left(z_{p}\right)_{p \in \mathbb{N}}$ approaching to $\partial D$ in terms of the far-field pattern.

In testing our inversion scheme, we simulate the inversion input data (far-field pattern) by solving the direct problem using the combined single- and double-layer potential method, see [8] and [9].

In the subsections 4.1 and 4.2 , we consider the first model, while subsection 4.3 is dedicated to a non-convex obstacle with mixed boundary conditions, from which we can test our theoretical results.

5.1. Reconstruction of $\partial D$. For the numerical test we take $\partial D=\{x=$ $\left.\left(x_{1}(t), x_{2}(t)\right)=1.2(\cos t, \sin t): t \in[0,2 \pi]\right\}$. We can construct $D_{a}^{p}$ in a special way by

$$
\partial D_{a}^{p}=\left\{\left(\tilde{x}_{1}(t), \tilde{x}_{2}(t)\right)=\left(1.2+\delta_{0}(p)\right)(\cos t, \sin t): t \in[0,2 \pi]\right\}
$$

and take $z_{p}=\left(\tilde{x}_{1}\left(t_{0}\right), \tilde{x}_{2}\left(t_{0}\right)\right)+\delta_{1}(p)\left(\cos t_{0}, \sin t_{0}\right)$ outside of $\bar{D}_{a}^{p}$ for $a=1.2\left(\cos t_{0}, \sin t_{0}\right)$ in $\partial D$. The smallness of $\delta_{1}(p)$ determines the singularity of $\Phi\left(x, z_{p}\right), \Phi_{\nu(a)}\left(x, z_{p}\right)$ on $\partial D_{a}^{p}$ near $z_{p}$.

Example 1. Construction of $\partial D$ with unknown impedance $\sigma(x)$ for given far-field data.

It follows from the first point of Theorem 3.1 that the boundary $\partial D$ can be reconstructed from the blow up behavior of the approximate values of the indicator function:

$$
I_{m, n}\left(z_{p}\right): \approx\left(\frac{\pi}{N}\right)^{2}\left|\sum_{i, j=1}^{2 N-1} \operatorname{Re}\left(\gamma_{2}^{-1} U^{\infty}(i, j) F_{m}^{p}(j) G_{m}^{p}(i)\right)\right|
$$

for large $m, n$, where $G_{n}^{p}(i):=g_{n}^{p}\left(\hat{x}_{i}\right), F_{m}^{p}(j):=f_{m}^{p}\left(\hat{d}_{j}\right)$ and $U^{\infty}(i, j)$ represents $u^{\infty}\left(-\hat{x}_{i}, d_{j}\right)$.

Therefore, we can test the numerical performance of this formula by taking $z_{p}$ approaching to $\partial D$. If $I_{m, n}\left(z_{p}\right)$ is greater than some a-priori given large value, for fixed $m, n$ large enough, we consider $z_{p}$ to be almost in $\partial D$. Notice that only the singularity in $\Phi_{\nu\left(\tilde{z}_{p}\right)}\left(x, z_{p}\right)$ is needed in this procedure, we do not need the boundary $\partial D$.

In our numerical implementations, we take $\kappa=0.6, N=32$ and keep the singularity of $\Phi\left(\cdot, z_{p}\right)$ unchanged near $\partial D_{a}^{p}$ by fixing $\delta_{1}=0.015$. When $z_{p}$ approaches to $\partial D$ by decreasing $\delta_{0}$ from different directions $t_{0}$, we get different values of $I_{m, n}\left(z_{p}\right)$. We choose the same constant $C B$ as the criterion for the blowing-up of the indicator 
$I_{m, n}\left(z_{p}\left(t_{0}\right)\right)$ at different direction $t_{0}$. That is, we choose $z_{p}\left(t_{0}\right)$ as the approximation of the point $a \in \partial D$ in the direction $t_{0}$ when $I_{m, n}\left(z_{p}\left(t_{0}\right)\right)$ is larger than $C B$. Then the constructed approximate position of $\partial D$ is given by interpolating those points. In this way, we can draw the approximate shape of $\partial D$ by choosing all directions $t_{0}$ using the same given $C B$. As obtained in the theoretical result, the larger the value $C B$ is, the better the approximation of $\partial D$ should be.

Consider the boundary reconstruction problem with non-constant impedance

$$
\sigma(x)=\frac{2+x_{1} x_{2}}{\left(3+x_{2}\right)^{2}}, \quad x \in \partial D
$$

Under this configuration, the reconstructions for different blowing-up criteria are shown in Figure 5.1 (left), while the distribution of indicator value is also given in the right picture.
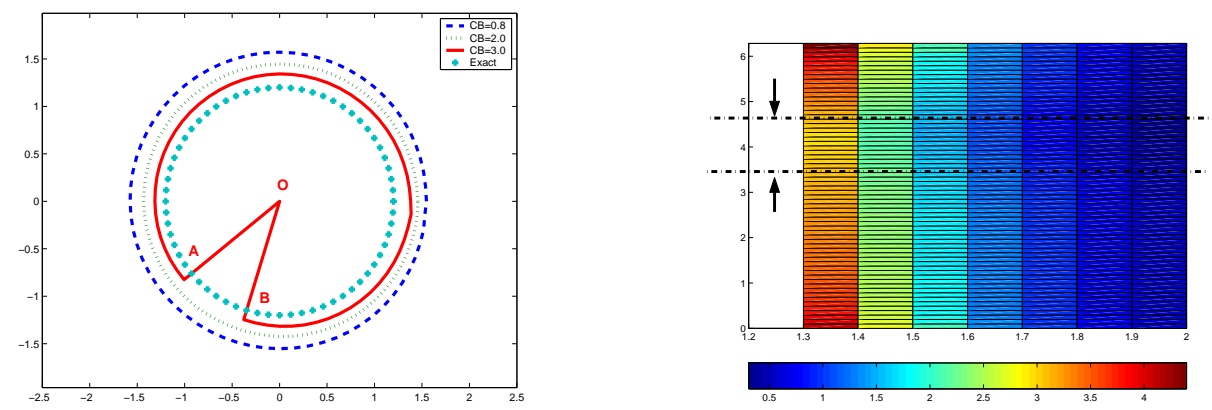

FIG. 5.1. Reconstruction of $\partial D$ for variable impedance with $\kappa=0.6$ with different blowing-up constants $C B$, the values of indicator are small in the directions corresponding to large impedance.

There is an interesting phenomena in the numerics. For a reasonable indicator value $C B=2$, we can see the whole rough shape of $\partial D$. However, for larger values, i.e. $C B=3$, most of the part of $\partial D$ can be seen with more satisfactory accuracy, but some part, i.e. $(\widehat{A B})$, is not visible. In this part the indicator value is less than 3 (but, of course, bigger than 2). This numerical performance is closely related to the impedance distribution in $\partial D$. It can be seen from the right part of Figure 5.1 that the indicator value is obviously smaller in the narrow domain at each radius layer. Therefore as $r$ decreases, the part of $\partial D$ related to these angles can not be detected with the same accuracy. Considering the distribution of boundary impedance, this part corresponds to $\sigma(x)$ with large value, so it can not be seen by using the same criterion values $C B$, as clearly as the other part. This may be explained by the fact that the scattered wave along these directions will be much absorbed. Another, but related, reason is the property $(3.19)$, i.e.

$$
\begin{gathered}
\lim _{m, n \rightarrow \infty} \operatorname{Re}\left[\gamma_{2}^{-1} \int_{S^{1}} \int_{S^{1}} u^{\infty}(-\hat{x}, d) f_{m}^{p}(d) g_{n}^{p}(\hat{x}) d s(\hat{x}) d s(d)\right]=\frac{ \pm 1}{4 \pi\left|\left(z_{p}-a\right) \cdot \nu(a)\right|}+ \\
+O\left(|\ln | z_{p}-a||\right)
\end{gathered}
$$

where $O\left(|\ln | z_{p}-a||\right)$ may be large if $\sigma$ is large near the point $a$. Hence numerically the second term can weaken the blowup of the first term.

Physically, this is the reason why we introduce the coated part of an obstacle, i.e. to avoid or perturb the detection of the obstacle. 
The other special property of this example is that the whole boundary shape can be well-reconstructed using only one blowing-up criterion $C B$. This comes from the special geometric shape and the fact that we have a complete impedance boundary condition. However, in the case of general problems, as for non-convex obstacle, with mixed boundary condition, we need to use multiple blowing-up values to get a satisfactory reconstruction, see subsection 4.3 .

5.2. Reconstruction of $\sigma(x)$ for known $\partial D$. For a given $a \in \partial D$, we take $z_{p} \in R^{2} \backslash \bar{D}_{a}^{p}$ with $D \subset \subset D_{a}^{p}$.

By the theoretical result given (3.21), the approximate formula for $\sigma(a)$ is

$$
\frac{\kappa}{\pi} \sigma(a) \approx \frac{1}{\left|\ln \left(\left(z_{p}-a\right) \cdot \nu(a)\right)\right|}\left(\frac{\pi}{n}\right)^{2} \sum_{i, j=1}^{2 n-1} \operatorname{Im}\left(\gamma_{2}^{-1} U^{\infty}(i, j) F_{m}^{p}(j) G_{n}^{p}(i)\right)
$$

for large $m, n$ and small $\left|z_{p}-a\right|$.

Example 2. Consider the variable impedance distribution given in Example 1.

We take $n=32$. First, let us keep the singularity unchanged by fixing $\delta_{1}$ and shrink the radius of $\partial D_{a}^{p}$ such that $z_{p} \rightarrow a \in \partial D$. The computed values of $\sigma(x(t))$ with fixed $\delta_{1}=0.015$ and different $\delta_{0}=1.22,0.82,0.12,0.02$ for two wave number $\kappa=0.6, \kappa=0.7$ are shown in Figure 5.2. It can be seen from this figure that when $\delta_{0} \rightarrow 0\left(\delta_{0}=0.02\right)$, the reconstruction results are satisfactory for both wave numbers.

It is interesting to see that when $\delta_{0}$ is large enough $\left(\delta_{0}=1.22\right)$, the reconstruction is invalid, even if here we use a strong singularity $\delta_{1}=0.015$. This is reasonable since the approximation to the strong singularity of the fundamental solution contain much error.
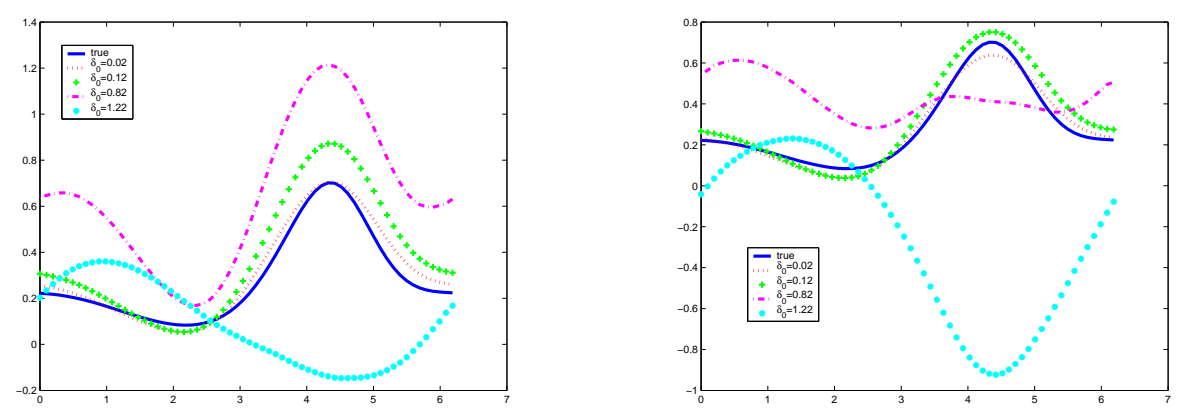

FIG. 5.2. Reconstruction of $\sigma(x)=\frac{2+x_{1} x_{2}}{\left(3+x_{2}\right)^{2}}$ for $\delta_{0}=1.22,0.82,0.12,0.02$ with two different wave numbers $\kappa=0.6$ (left) and $\kappa=0.7$ (right), where we fix $\delta_{1}=0.015$.

Finally, we fix $\partial D_{a}^{p}$ near $\partial D$ and take $z_{p}$ tending to $\partial D_{a}^{p}$. In our configuration, this means $\delta_{1} \rightarrow 0$ for fixed small $\delta_{0}$. The tests with $\delta_{0}=0.002$ for two wave number $\kappa=0.6,1.0$ at different $\delta_{1}=0.025,0.020,0.015,0.010$ are given in Figure 5.3, which show the influence of the singularity. It can be seen that the approximation is sensitive to the wave number $\kappa$. The reason is that we ignore the remained term $C /|\ln |\left(z_{p}-a\right) \cdot \nu(a)||$, where the constant $C$ comes from (4.5). Theoretically, this terms tend to 0 as $z_{p} \rightarrow a$. However, this procedure causes a difficulty in approximating the fundamental solution. We expect that the constant $C$ becomes large as $\kappa$ becomes small. This is naturally related to the following relation, in the 2-dimensional, case between the fundamental solution to the Helmholtz and Laplace equations:

$$
H_{0}^{(1)}(\kappa|x-y|)=-\frac{1}{2 \pi} \ln (|x-y|)-\ln \kappa+O(1)
$$


locally for $x, y \in R^{2}$ and $\kappa$ small enough, see [8]. This implies that the difference between the fundamental solution behaves as $\ln \kappa$. So the constant $C$ should have a similar behavior with respect to $\kappa$. These remarks on the dependency on wave number $\kappa$ are also observed in the tests for detecting $\partial D$. However, we think that this is just a $2 \mathrm{D}$ phenomenon.
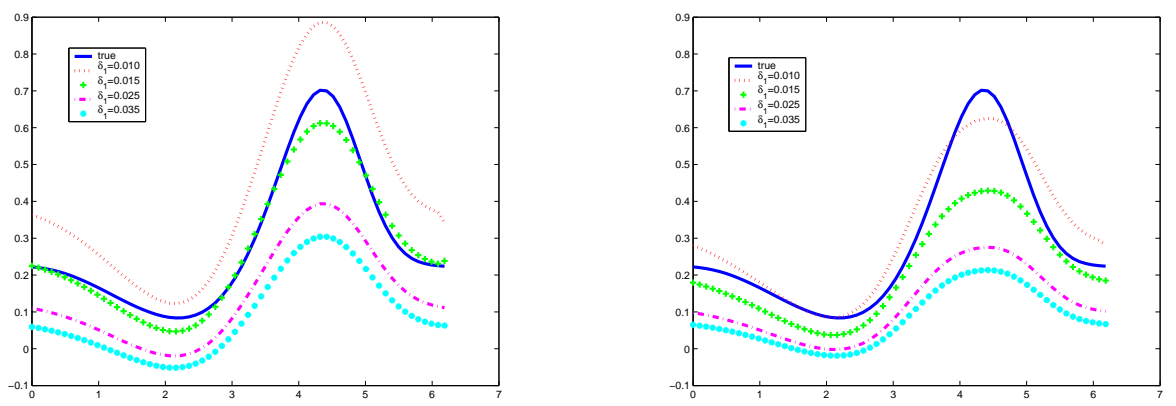

FIG. 5.3. Reconstruction of $\sigma(x)=\frac{2+x_{1} x_{2}}{\left(3+x_{2}\right)^{2}}$ for $\delta_{1}=0.010,0.015,0.025,0.035$ with two different wave numbers $\kappa=0.6$ (left) and $\kappa=1.0$ (right), where we fix $\delta_{0}=0.002$.

5.3. Reconstruction of an obstacle with mixed type boundary. Since the main advantage of our inversion method is its ability to identify the full complex obstacle simultaneously, here we consider the numerical behavior of our inversion method acting on a non-convex obstacle with mixed boundary condition.

Example 3. Consider a non-convex obstacle $D$ with the boundary

$$
\partial D=\left\{x: x(t)=\left(x_{1}(t), x_{2}(t)\right)=(\cos t+0.65 \cos 2 t-0.65,1.5 \sin t), t \in[0,2 \pi]\right\} .
$$

Let $\partial D$ be composed of sound-soft part $\partial D_{D}$ for $t \in[0,1.42 \pi]$ and impedance part $\partial D_{I}$ for $t \in[1.42 \pi, 2 \pi]$. In $\partial D_{I}$, we assume the impedance coefficient $\sigma(x(t)) \equiv 3$.

We also choose $\partial D_{a}^{p}$ and $z_{p}$ in a special way. For two small constants $\delta_{0}, \delta_{1}>0$, we take

$$
\left\{\begin{array}{l}
\partial D_{a}^{p}=\left\{y(t):=x(t)+\delta_{0} \times(\cos t, \sin t): x(t) \in \partial D, t \in[0,2 \pi]\right\} \\
z_{p}(t)=y(t)+\delta_{1} \times(\cos t, \sin t) \text { for } t \in[0,2 \pi]
\end{array}\right.
$$

In terms of Theorem 3.1, the inversion schemes contain the following three steps:

Step 1. Identify the location of $\partial D$ using (3.18);

Step 2. Distinguish the different parts of $\partial D$ in terms of (3.20);

Step 3. Reconstruction of $\sigma(x)$ in $\partial D_{I}$ from (3.21).

We present the numerical results with fixed wave number $\kappa=0.9$ and $\delta_{1}=0.01$.

Step 1 . we take $n=16$ and decrease $\delta_{0}=l \times 0.05$ by taking $l$ from 20 to 2 . The indicator values in (3.18) for $\partial D$ are computed for $z_{p}(t)$ and $\partial D_{a}^{p}$ specified here for every direction $t_{j}$ and different $\delta_{0}$. Then we draw the contour line to obtain an approximation of $\partial D$. As for example 1 and example 2, we choose some appropriate value $C B$ for the stopping rule of $l$. In the case if the indicator is always less than $C B$ in some direction, we take $z_{p}$ for the initial guess (the largest $l$ ) as an approximate location of points in $\partial D$.

In this case, the situation is different from the examples given in the previous subsections, the reconstruction of the boundary using only one blowing-up value $C B$ 
is not sufficient, see the left picture in Figure 5.4 for the reconstruction of $C B=3.0$, where the kite-shape in red color is the exact obstacle. Enlarging $C B$ can improve the reconstruction along some directions, but the approximation to the whole boundary is still not satisfactory. The reason for this phenomena is that our theoretical results do not guarantee the uniform blowing-up property for all directions.
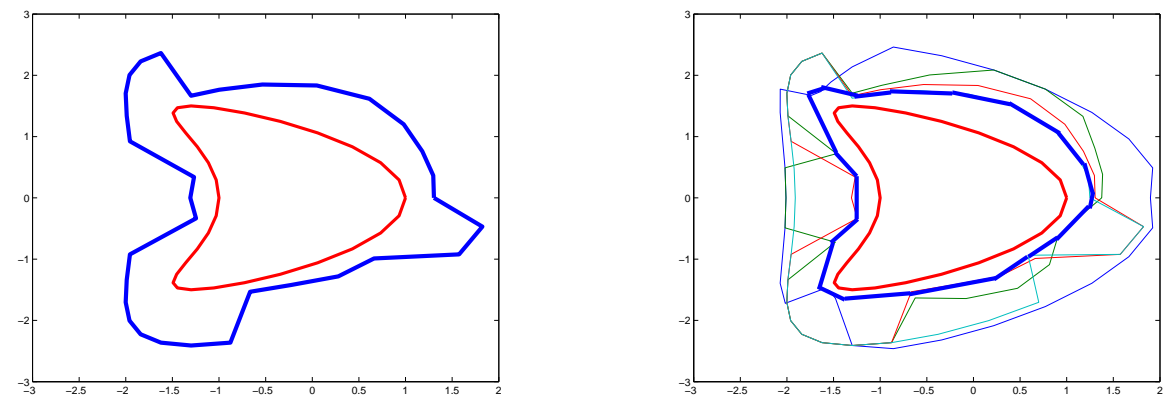

FIG. 5.4. Reconstruction of $\partial D$ with $C B=3.0$ (left) and $4 C B^{\prime} s$ (right) by concave-hull.
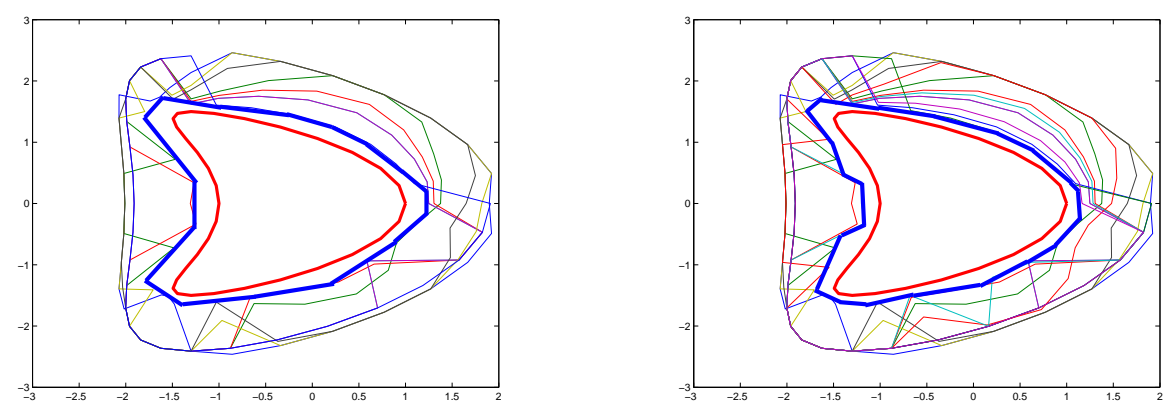

FIG. 5.5. Reconstruction of $\partial D$ by concave-hull using $8 C B^{\prime} s$ (left) and 12 $C B^{\prime} s$ (right).

To overcome this difficulty and apply our reconstruction formula, we propose to combine the reconstruction results for different $C B$ together and take the concave hull. Then $\partial D$ can be approximated very well, see Figure 5.4 where we compare the shape given by using one $\mathrm{CB}$ only and the one obtained by using and $4 C B$ values. In Figure 5.5, we show the reconstruction results by using eight and twelve $C B$ values. Precisely, the reconstructions in Figure 5.4 and Figure 5.5 correspond the following $C B^{\prime} s$ values:

Figure 5.4 (Right): 4 values $-C B=0.8,2.5,3.0,3.5$;

Figure 5.5 (Left): 8 values $-C B=0.8,2.5,3.0,3.5,1.2,1.5,5.5,6.5$;

Figure 5.5 (Right): 12 values- $C B=0.8,2.5,3.0,3.5,1.2,1.5,5.5,6.5,2.0,3.2,4.5,6.0$.

It can be seen that the reconstruction is satisfactory. This means that using the technic of combining several CB's, the theoretical formulas provide good reconstructions.

We give the indicator value distribution in Figure 5.6 for all directions $t$ with different $l$ at each direction. We can see how the indicator near $t=0.58 \pi, \pi, 1.42 \pi$ has some special property, which explains the difficulty of reconstructing these parts shown in Figure 5.4 and Figure 5.5.

Next we consider Step 2 and Step 3 by using (3.20) and (3.21), that is, test the numerical behavior in distinguishing the boundary type and impedance in $\partial D_{I}$. 


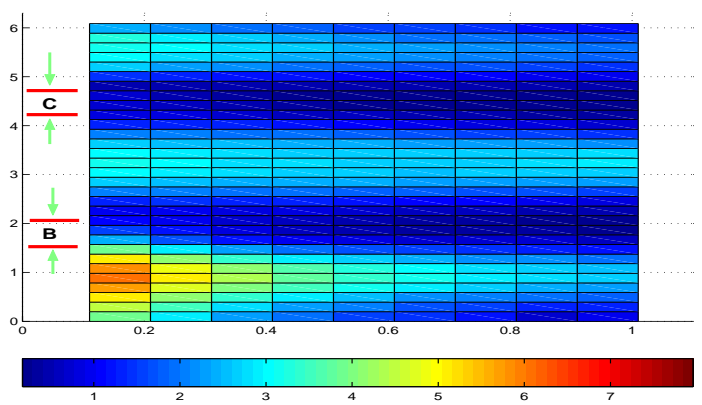

FIG. 5.6. The indicator values for boundary shape.

Different from the formula (3.18), these two formulas need the boundary shape $\partial D$ which is theoretically obtained from (3.18). Since we can only get some approximation to $\partial D$ numerically in terms of (3.18), it is necessary to check the approximate versions of (3.20) and (3.21) for distinguishing the boundary type and recovering $\sigma(x)$ on the coating part.

Step 2. We express the quantitative behavior for distinguishing $\partial D_{D}$ and $\partial D_{I}$ by given the indicator distribution. As explained in Step 1, by using different blowing-up criterion in the shape reconstruction, we can get a good approximation to $\partial D$. In this step, we specify $\partial \tilde{D} \approx \partial D$ with an explicit expression given by

$$
\partial \tilde{D}=\left(\begin{array}{cr}
\cos \delta^{*} & -\sin \delta^{*} \\
\sin \delta^{*} & \cos \delta^{*}
\end{array}\right)\left[\partial D+\left\{\delta^{*} \times(\cos t, \sin t): t \in[0,2 \pi]\right\}\right]
$$

with small constant $\delta^{*}>0$, for simplicity. In this way, $\tilde{D}$ is no more symmetric with respect to $x_{1}$ and the location for corner part also differs from that of $\partial D$. To check the effect of this domain approximation on (3.20) and (3.21), we also evaluate them using the exact $\partial D$.

We generate $\left(\partial \tilde{D}_{a}^{p}, \partial D_{a}^{p}\right)$ from $(\partial \tilde{D}, \partial D)$ and therefore the sequences $\left(\left\{\tilde{z}_{p}\right\},\left\{z_{p}\right\}\right)$ as in (5.3). In this way, we have $\tilde{z}_{p} \rightarrow \tilde{a} \in \partial \tilde{D}, z_{p} \rightarrow a \in \partial D$ and $\delta_{0}, \delta_{1} \rightarrow 0$. In the computation, we take $n=32$ and decrease $\delta_{0}=l \times 0.05 \rightarrow 0$ by taking $l$ from 10 to 2 . The indicator behavior using the same far-field pattern for $\partial D$ and $\partial \tilde{D}$ with $\delta^{*}=0.05$ are given in Figure 5.7.
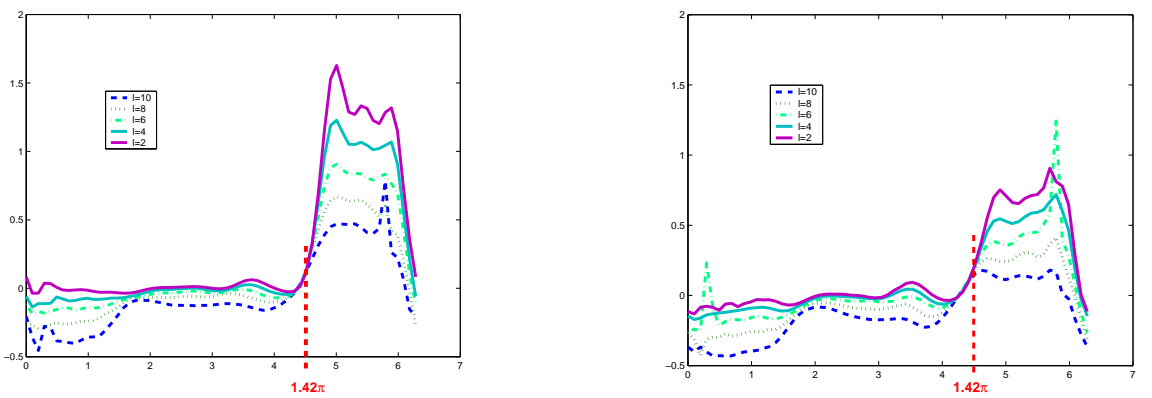

FIG. 5.7. Indicator values for different part of the boundary using exact $\partial D$ (left) and $\partial \tilde{D}$ with $\delta^{*}=0.1$ (right).

Noticing the fact that the sound-soft part corresponds to the parameter $t \in$ $[0,1.42 \pi]$, while the impedance part is related to $t \in[1.42 \pi, 2 \pi]$, the above numerical 
behavior supports (3.21) strongly with a large difference in $[0,1.42 \pi]$ and $[1.42 \pi, 2 \pi]$, that is, we can distinguish the boundary type in terms of the obvious difference of indicator values when $z_{p}$ approaches to the boundary (for small $l$ ), even if the boundary shape is known with a relative error.

Step 3. We compute the impedance coefficient on $\partial D_{I}$ by applying the formulas for $\partial D$ and also for its approximation $\partial \tilde{D}$, respectively. The reconstruction behavior for exact $\partial D$ as well as its approximation $\partial \tilde{D}$ with $\delta^{*}=0.05$ is shown in Figure 5.8. It can be seen that, for given exact boundary shape $\partial D$, the theoretical result (3.21) for the impedance is valid (left figure), except near the end points of $\partial D_{I}$. The rough approximation in this part is reasonable, since this part is near to the sound-soft boundary. Using the approximate domain $\partial \tilde{D}$, the impedance can still be captured (right figure). Of course, the reconstruction is less accurate. Due to the nonconvex property of the obstacle and the mixed boundary condition, we think these results are satisfactory. However, if the perturbation for $\partial D$ is very large, then the
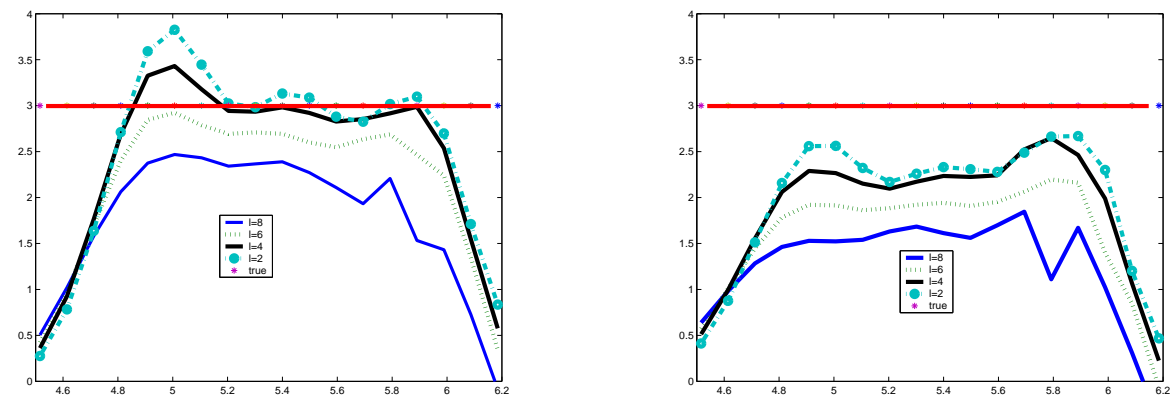

FIG. 5.8. Recovery of $\sigma$ using exact $\partial D$ (left) and approximate $\partial \tilde{D}$ with $\delta^{*}=0.05$ (right).

reconstruction of $\sigma(x(t))$ is much contaminated. The reason is due to the sensitivity of the approximate reconstruction to the boundary shape, especially for the corner part of the non-convex domain, noticing that in the formula (3.21), the normal direction $\nu(a)$ appears. The perturbation scheme (5.4) moves the position of the corner part by rotation. An inversion result for the impedance with $\delta^{*}=0.1$ is shown in Figure 5.9. From the left picture of this figure, we see how the corner part of $\partial \tilde{D}$ with $\delta^{*}=0.1$ has been much moved from that of $\partial D$ with a relative error almost $10 \%$.
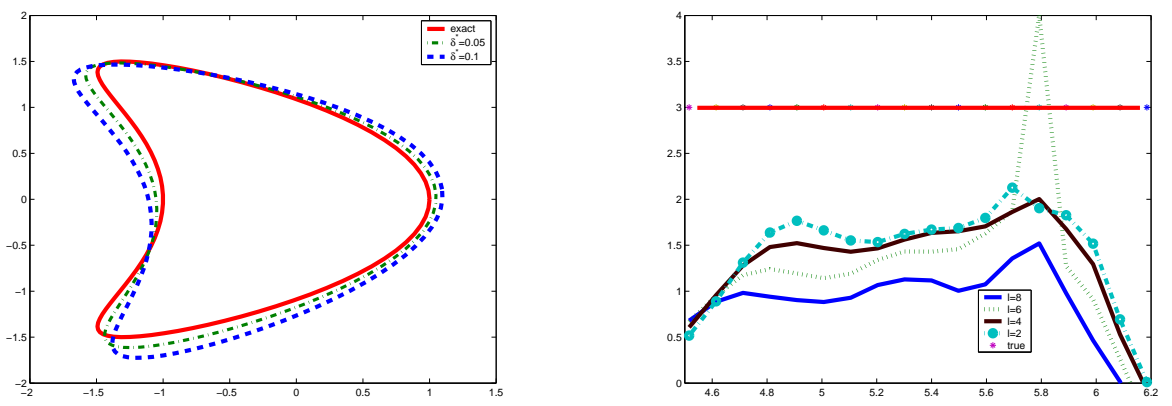

FIG. 5.9. Approximate $\partial \tilde{D}$ with $\delta^{*}=0.05,0.1$ (left) and reconstruction of $\sigma$ for different $l$ from approximate $\partial \tilde{D}$ with $\delta^{*}=0.1$ (right).

We conclude that the theoretical results (3.20) and (3.21) are well supported by our numerical tests even for non-convex domains. If the approximate domain $\partial \tilde{D}$ 
is used in these two formulas, then we can still distinguish the boundary type in terms of the obvious blowing-up property of the indicator. However, the quantitative identification of impedance depends on the error level of $\partial D$.

Acknowledgments. The authors thank the anonymous referees for their valuable comments and suggestions, which led to a modified version of this paper. The first author is supported by NSFC (No. 10371018). The second author is partially supported by Grant-in-Aid for Scientific research (B)(2) (N0.14340038) of Japan Society for Promotion of Science. The third author is supported by IIRC of Kyung Hee University, Korea, via the SRC/ERC program of MOST/KOSEF (R11-2002-103).

\section{REFERENCES}

[1] I. AKDUMAN AND R. KRESS, Direct and inverse scattering problems for inhomogeneous impedance cylinders of arbitrary shape, Radio Science, Vol.38, No.3, (2003), pp. 1055-1064.

[2] F. CAKoni, D. Colton, The determination of the surface impedance of a partially coated obstacle from far field data, SIAM. J. Appl. Math., Vol.64,(2004), 709-723.

[3] F. Cakoni, D. Colton, P. Monk, The determination of the surface conductivity of a partially coated dielectric, SIAM. J. Appl. Math., Vol.65, No.3, (2005), pp. 767-789.

[4] F. Cakoni, D. Colton, Qualitative Methods in Inverse scattering Theory, Interaction of Mechanics and Mathematics, Springer, 2006.

[5] J. Cheng, J. J. LiU, G. Nakamura and S. Z. Wang, Recovery of boundaries and types for multiple obstacles from the far-field pattern, to appear in Quart. Applied Maths.

[6] J. Cheng, J. J. LiU, G. Nakamura, Recovery of the shape of an obstacle and the boundary impedance from the far-field pattern, J. of Maths of Kyoto University, Vol.43, No.1, (2003), pp. $165-186$

[7] D. Colton And A. KIRsch, A simple method for solving inverse scattering problems in the resonance region, Inverse Problems, Vol.12, (1996), pp. 383-393.

[8] D. Colton, R. Kress, Inverse Acoustic and Electromagnetic Scattering Theory, 2nd edition, Berlin-Springer, 1998.

[9] D. Colton, R. Kress, Integral Equation Methods in Scattering theory, John Wiley, New York, 1983.

[10] D. J. Hoppe AND Y. RAhmat-SAmi, Impedance boundary condition in electromagnetism, Taylor and Francis, 1995.

[11] N. Honda, G. Nakamura, R. Potthast And M. Sini, The no-response approach and its relation to non-iterative methods for the inverse scattering, Annali. Math. Pur. Appl. Appeared online.

[12] M. Gruter and K. O. Widman, The Green function for uniformly elliptic equations, Manuscripta math, Vol.37, (1982), pp. 303-342.

[13] M. IKenAtA, Reconstruction of the shape of the inclusion by boundary measurements, Comm. PDE, Vol.23, (1998), pp. 1459-1474.

[14] M. Ikehata, Reconstruction of obstacles from boundary measurements, Wave Motion, Vol.3, (1999), pp. 205-223.

[15] M. IkehatA, A new formulation of the probe method and related problems, Inverse Problems 21, no. 1, (2005), pp. 413-426.

[16] A. KIRSCH, Characterization of the shape of a scattering obstacle using the spectral data of the far field operator, Inverse Problems, Vol.14, (1998), pp. 1489-1512.

[17] R. KRess AND W. Rundell, Inverse scattering for shape and impedance, Inverse Problems, Vol.17, (2001), pp. 1075-1085.

[18] W. Littman, G. Stanpacchia And H. F. Weinberger, Regular points for elliptic equations with discontinuous coefficients, Ann. Scuola Norm. Sup. Pisa (III), 17, (1963), pp. 43-77.

[19] G. Nakamura, M. Sini, Pointwise determination of the surface impedance from scattering data, Preprint.

[20] G. Nakamura, R. Potthast and M. Sini, Unification of the probe and singular sources methods for the inverse boundary value problem by the non-responce test, Comm. PDE, Vol. 31, No. 10, (2006), pp. 1505-1528.

[21] R. Potthast, Point Sources and Multipoles in Inverse Scattering Theory, Research Notes in Mathematics, Vol.427, Chapman-Hall/CRC, Boca Raton, Fl, 2001. 
[22] R. Роттнаsт, Sampling and probe methods - an algorithmical view, Computing, Vol.75, No.2-3, (2005), 215-235.

[23] R. Роттнаsт, A survey on sampling and probe methods for inverse problems, Inverse Problems, Vol.22, No.2, (2006), R1-R47.

[24] V. A. Solonnikov, On Green's matrices for elliptic boundary value problems(I), Proc. Steklov. Inst. Math,, Vol.110, 1970.

[25] V. A. Solonnikov, The Green's matrices for elliptic boundary value problems(II), Boundary Value Problems of Mathematical Physics, 7, Trudy. Math. Inst. Steklov., Vol.116, (1971), pp. 181-216. 